\title{
CLASSIFICATION OF SYMMETRY GROUPS FOR PLANAR $n$-BODY CHOREOGRAPHIES
}

\author{
JAMES MONTALDI and KATRINA STECKLES \\ School of Mathematics, University of Manchester, Manchester M13 9PL, UK; \\ email: j.montaldi@manchester.ac.uk
}

Received 15 May 2013; accepted 12 November 2013

\begin{abstract}
Since the foundational work of Chenciner and Montgomery in 2000 there has been a great deal of interest in choreographic solutions of the $n$-body problem: periodic motions where the $n$ bodies all follow one another at regular intervals along a closed path. The principal approach combines variational methods with symmetry properties. In this paper, we give a systematic treatment of the symmetry aspect. In the first part, we classify all possible symmetry groups of planar $n$-body collision-free choreographies. These symmetry groups fall into two infinite families and, if $n$ is odd, three exceptional groups. In the second part, we develop the equivariant fundamental group and use it to determine the topology of the space of loops with a given symmetry, which we show is related to certain cosets of the pure braid group in the full braid group, and to centralizers of elements of the corresponding coset. In particular, we refine the symmetry classification by classifying the connected components of the set of loops with any given symmetry. This leads to the existence of many new choreographies in $n$-body systems governed by a strong force potential.
\end{abstract}

2010 Mathematics Subject Classification: 37C80, 70F10 (primary); 58E40 (secondary)

\section{Introduction}

The problem of determining the motion of $n$ particles under gravitational interaction has long been of interest, and since Poincaré there has been particular interest in periodic motions. In the last 20 years, renewed interest has followed the discovery of what are now called choreographies: periodic motions where the particles, assumed to be of equal mass, follow each other around a closed path at regular intervals. In 1993, Moore [31] discovered the first of these, where three identical particles move along a figure- 8 curve; he found this 

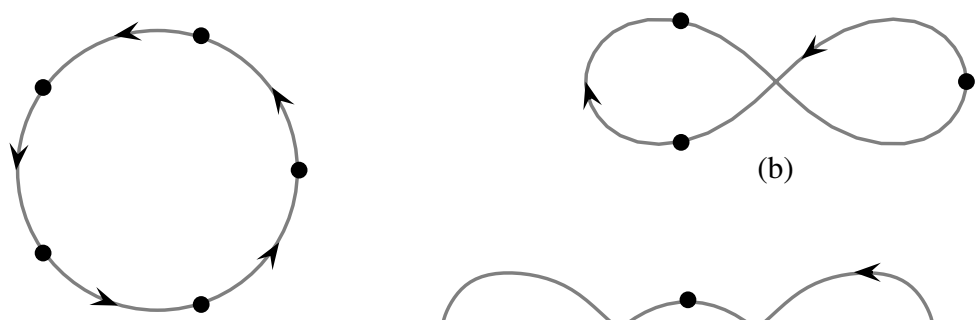

(b)

(a)
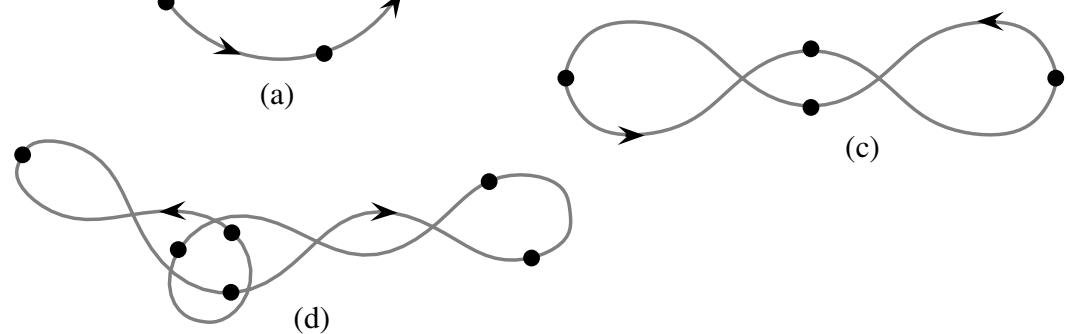

(c)

Figure 1. Examples of planar choreographies; (a) circular, (b) the Figure 8, (c) the super-8, and (d) a nonsymmetric choreography.

numerically. Independently, Chenciner and Montgomery [8] (re)discovered this figure- 8 solution a few years later, but they proved its existence using a clever combination of symmetry methods and variational techniques.

Since the work of Moore, Chenciner, and Montgomery, there have been many papers written on the subject of choreographies. We restrict ourselves to the planar case, although interesting examples of choreographies have been shown to exist in higher dimensions $[1,9,12,15]$. In the plane, the first choreography known (in hindsight) was the circular choreography of Lagrange, in which the particles are positioned at the vertices of a regular $n$-gon rotating with constant speed about its centre. Soon after the work of Chenciner and Montgomery, J. Gerver suggested a four-particle choreography on what is called the 'super-8', a curve similar to a figure- 8 but with three internal regions and two crossings ([7, p. 289] and Figure 1(c)). The papers [7,35] contains many examples of choreographies, found numerically, and it will be noticed that almost all have some geometric symmetry.

Some work approaches the questions using numerics and some uses an analytic-topological approach, but almost all methods use a variational setting for the problem. The original paper by Moore [31] was asking how the theory of braids could be used in the study of dynamical systems of $n$ interacting bodies in the plane-any periodic motion of $n$ particles can be represented by a braid (indeed a pure braid, as the particles return to their original position after one period), and Moore's numerical approach was to use the braid as an 'initial condition' for the variational problem and then 'relax' the curve by decreasing 
the action. Chenciner and Montgomery's approach was also variational, but they used explicitly the symmetries involved in the figure- 8 solution, together with the variational set-up, and the crux of their existence proof was to show that minimizing the action within the given symmetry class did not involve collisions.

The idea of using symmetry methods in the variational problem was taken up in a very interesting paper by Ferrario and Terracini [13], where they gave, among other things, conditions on the symmetry under consideration guaranteeing that a minimizer of the action is free of collisions (their 'rotating circle' condition, which we describe in Section 2).

An excellent review by Terracini was published in 2006 [39], containing many more references.

The principal aim of the present paper is to make systematic the combination of topological (braid) methods and symmetry methods. We begin by classifying all possible symmetry groups arising for (collision-free) choreographies in the plane, and then proceed to study symmetries in loop space, first in general and then referring specifically to choreographies. The work is an extension of the work presented in the second author's thesis [37].

1.1. Configurations and symmetries. We are interested in the motion of an isolated system of $n$ identical particles in the plane. We identify the plane with $\mathbb{C}$, the complex numbers. Under these assumptions, the centre of mass of the particles is given by $(1 / n) \sum_{j} z_{j}$, and without loss of generality we can take this point to be fixed at the origin. In addition, we assume that the particles do not collide. Later, we assume that they interact under a conservative attractive force. Much of this section follows the work of Ferrario and Terracini [13].

The configuration space of the system is therefore

$$
X^{(n)}:=\left\{\left(z_{1}, \ldots, z_{n}\right) \in \mathbb{C}^{n} \mid \sum_{j} z_{j}=0, z_{i} \neq z_{j} \forall i \neq j\right\},
$$

which is a (noncompact) manifold of real dimension $2 n-2$. There is a natural symmetry group acting on $X^{(n)}$, namely the product of the orthogonal group in the plane and the group of permutations of the $n$ points $\Gamma:=\mathrm{O}(2) \times S_{n}$, acting by

$$
(A, \sigma) \cdot\left(z_{1}, \ldots, z_{n}\right)=\left(A z_{\sigma^{-1}(1)}, \ldots, A z_{\sigma^{-1}(n)}\right) .
$$

All group actions will be left actions, whence the inverse on the permutation in (1.1).

Let $\Lambda=\Lambda X^{(n)}$ be the space of all loops in $X^{(n)}$. A loop is by definition a continuous map

$$
u: \mathbb{T} \rightarrow X^{(n)},
$$


where $\mathbb{T}$ is the time circle: we identify $\mathbb{T}=\mathbb{R} / \mathbb{Z}$, so loops are parameterized by $t \in[0,1]$. Then

$$
u(t)=\left(z_{1}(t), \ldots, z_{n}(t)\right) \in X^{(n)},
$$

where each $z_{j}: \mathbb{T} \rightarrow \mathbb{C}$.

Denote by $\widehat{S}^{1}$ the symmetry group of rotations and reflections of the time circle $\mathbb{T}$, which is isomorphic to $\mathrm{O}(2)$. The action of $\Gamma$ on $X^{(n)}$ extends to an action of $\Gamma \times \widehat{\mathrm{S}^{1}}$ on the loop space $\Lambda$ : if $\tau \in \widehat{\mathrm{S}}^{1}$, we put

$$
((A, \sigma, \tau) \cdot u)(t):=(A, \sigma) \cdot u\left(\tau^{-1}(t)\right),
$$

where $(A, \sigma)$ acts as in (1.1). A loop $u$ is said to have symmetry $G<\Gamma \times \widehat{\mathrm{S}^{1}}$

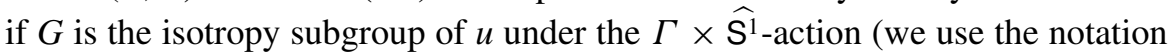
$G<H$ to mean that $G$ is a subgroup of $H$ ). Explicitly, this means that

$$
u(\tau(t))=(A, \sigma) \cdot u(t), \quad \forall(A, \sigma, \tau) \in G .
$$

Notice that $g=(I, \sigma, \tau) \in G$ means that

$$
z_{\sigma(j)}(\tau(t))=z_{j}(t) \quad(\forall j, t),
$$

and consequently particles whose labels are within the same orbit of $\sigma$ follow the same path. In particular, if $\sigma$ is a cycle of order $n$ (the number of particles), then all the particles follow the same path.

A particular subgroup of $\Gamma \times \widehat{\mathrm{S}^{1}}$ of central interest is the choreography group $\mathfrak{C}_{n}$, which is the cyclic group of order $n$ generated by $\mathfrak{c}=\left(I, \sigma_{1},-1 / n\right)$, where $\sigma_{1}$ is the cycle $\sigma_{1}=(123 \ldots n) \in S_{n}$.

Recall that, given any action of a group $G$ on a space $X$, the fixed point space is defined to be

$$
\operatorname{Fix}(G, X)=\{x \in X \mid \forall g \in G, g \cdot x=x\} .
$$

DEFINITION 1.1. A choreography is an element of the fixed point space $\operatorname{Fix}\left(\mathfrak{C}_{n}, \Lambda X^{(n)}\right)$.

We denote this fixed point space by $\Lambda^{\mathfrak{c}}=\Lambda^{\mathrm{c}} X^{(n)}$. Explicitly, the loop (1.2) is a choreography if, for each $j=1, \ldots, n$,

$$
z_{j+1}(t)=z_{j}\left(t+\frac{1}{n}\right),
$$

where the index is taken modulo $n$; in particular, particle 1 follows particle 2, which in turn follows particle 3 , and so on, and all with the same time delay of $1 / n$. As already pointed out, this definition requires all the particles to move on the same curve. Such motions are sometimes called simple choreographies, to 
distinguish them from more general choreographies where more than one curve is involved, and possibly different numbers of particles on different curves: we only consider these simple choreographies. Note that the definition does not imply that the particles are in numerical order around the curve, as Example 1.3 below shows.

It follows from the definition that the symmetry group $G$ of any choreography satisfies $\mathfrak{C}_{n}<G$ (in Proposition 2.1 we show that it is in fact a normal subgroup). Since $\mathfrak{C}_{n}$ is of order $n$, it follows that $n$ divides the order of the symmetry group $G$ of any choreography.

For a given symmetry group $G<\Gamma \times \widehat{\mathrm{S}^{1}}$, we denote by $\rho, \sigma, \tau$ the projections of $G$ to each component. That is, given an element $g \in \Gamma \times \widehat{\mathrm{S}^{1}}$, we write its three components as $\rho(g) \in \mathrm{O}(2), \sigma(g) \in S_{n}$, and $\tau(g) \in \widehat{\mathrm{S}^{1}}$.

DEFINITION 1.2. A subgroup $G<\Gamma$ is said to be nonreversing if $\tau(G)<\mathrm{S}^{1}$; otherwise it is reversing.

These are what Ferrario and Terracini call symmetry groups of cyclic and dihedral type, respectively [13]. Note that their 'brake type' symmetry groups cannot occur in collision-free (simple) choreographies with more than one particle.

EXAMPLE 1.3. One important—but in a sense trivial—class of choreography is what we call the circular choreographies, where the particles lie at the vertices of a regular $n$-gon which rotates uniformly about the centre of mass; they are the generalizations of the Lagrange solution to $n$ particles. Explicitly, consider the parameterized circle $z(t)=\exp (2 \pi i t)$ in the plane. Let $\ell$ be an integer coprime to $n$, and define a motion of $n$ particles by, for $j=1, \ldots, n$,

$$
z_{j}(t)=e^{2 \pi i \ell j / n} z(\ell t) \text {. }
$$

This is easily seen to be a choreography, satisfying (1.4). The full symmetry group of this motion is isomorphic to a semidirect product $\mathrm{O}(2) \ltimes \mathbb{Z}_{n} \simeq$ $\left(\mathrm{SO}(2) \times \mathbb{Z}_{n}\right) \rtimes \mathbb{Z}_{2}$; an explicit description of the elements of this group is given in Equation (2.1). In this motion, particle $j$ immediately follows particle $j+m$ around the circle, where $m \ell=1(\bmod n)$, with a time delay of $1 / \ell m$; that is,

$$
z_{j+m}(t)=z_{j}\left(t+\frac{1}{m \ell}\right),
$$

which implies the choreography condition (1.4). In this example, we have

$$
\operatorname{ker} \rho=\mathfrak{C}_{n}, \quad \operatorname{ker} \sigma \simeq \mathrm{SO}(2), \quad \operatorname{ker} \tau=\left\langle\left(R_{2 \pi \ell / n}, \sigma_{1}, 0\right)\right\rangle \simeq \mathbb{Z}_{n} .
$$

We assume that $\ell$ is coprime to $n$, for otherwise this motion involves particles coinciding for all time. 
Classification. The main result of the first part of the paper (stated as Theorem 2.3) is a complete classification of all possible symmetry groups of (simple) planar choreographies. In other words, we classify all those subgroups of $\mathrm{O}(2) \times S_{n} \times \widehat{\mathrm{S}}^{1}$ that on the one hand contain $\mathfrak{C}_{n}$ and on the other are realized as the symmetry group of some collision-free $n$-body motion. For a given number $n$ of particles, one finds that there are two infinite families of symmetry group and, if $n$ is odd, three exceptional symmetry groups. Full details of the symmetry groups are given in Section 2; here, we give a brief description. For the infinite families, the curve on which the particles move has the symmetry of a regular $k$-gon for some $k \geq 1$. As the particles move, they visit the 'vertices' of the $k$-gon in some order. This order is similar to the difference between a pentagon and a pentagram: in the former the vertices are visited in geometric order, while in the latter the vertices are visited alternately (that is, in the order 1, 3, 5, 2, 4, rather than $1,2,3,4,5)$. There is a convenient notation used to distinguish these, the so-called Schläfli symbol. In this notation, the basic regular convex $k$-gon is denoted $\{k\}$, while the $k$-gon with every $\ell$ th vertex visited in sequence is denoted $\{k / \ell\}$-in particular, $\{k / 1\}=\{k\}$. Thus the pentagon is denoted $\{5\}$, while the pentagram is denoted $\{5 / 2\}$. In order for the geometric object $\{k / \ell\}$ to consist of a single closed curve, it is necessary and sufficient that $k$ and $\ell$ are coprime (which we write throughout as $(k, \ell)=1$ ). We adapt this notation, and denote the symmetry groups for $n$ particles moving on a curve of type $\{k\}$ or $\{k / \ell\}$ by $C(n, k)$ or $C(n, k / \ell)$ respectively if there is no time-reversing symmetry, and by $D(n, k)$ or $D(n, k / \ell)$ respectively if there is such a symmetry. See for example Figure 3 for choreographies illustrating the difference between $D(6,5)$ (pentagon) and $D(6,5 / 2)$ (pentagram). In these infinite families, the time-reversing symmetries occur in combination with a reflection in the plane. As $k \rightarrow \infty$, the $k$-gon tends to a circle, and so we denote the symmetry of the circular choreography introduced above by $D(n, \infty / \ell)$.

On the other hand, if $n$ is odd, there are three exceptional symmetry groups, denoted $C^{\prime}(n, 2), D^{\prime}(n, 1)$, and $D^{\prime}(n, 2)$ (where, as always, $n$ is the number of particles). For example, the choreography in Figure 8 has symmetry $D^{\prime}(3,2)$. In these groups there is always an element which either acts as time-reversing symmetry but not a reflection (in $D^{\prime}(n, 1)$ ), or a reflection which is not acting as time-reversal, in $C^{\prime}(n, 2)$, or both, in the case of $D^{\prime}(n, 2)$. Precise details are given in Section 2, while a number of different choreographies are illustrated in Figure 2.

Comparison to recent literature. Stewart [38] gives a classification of symmetry groups arising in many-body problems which is different from ours. This difference arises for two reasons: first, Stewart does not restrict attention to 


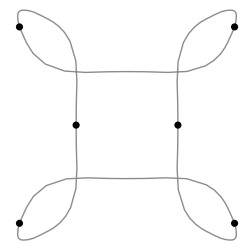

(a) $D(6,4)$

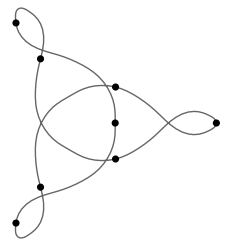

(e) $D(8,3)$

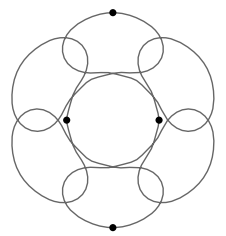

(i) $D(4,6)$

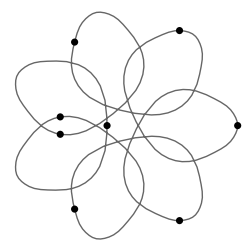

(m) $D(8,7)$

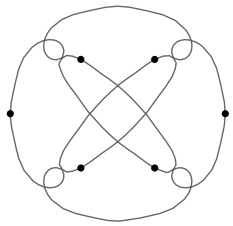

(b) $D(6,4)$

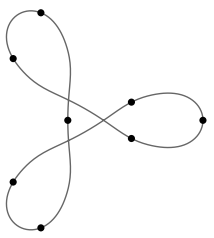

(f) $D(8,3)$

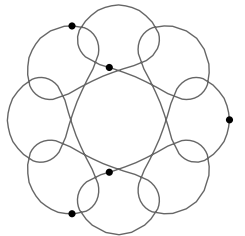

(j) $D(5,8)$

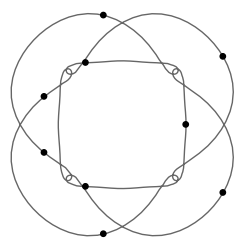

(n) $D(9,4)$

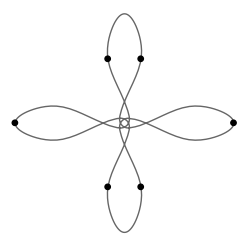

(c) $D(6,4)$

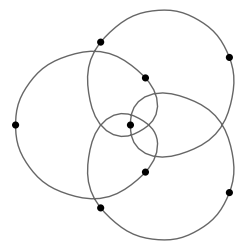

(g) $D(8,3)$

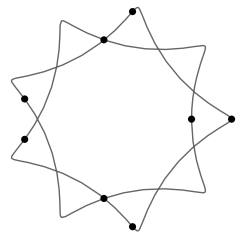

(k) $D(8,9 / 2)$

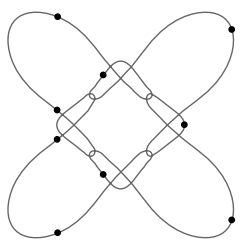

(o) $D(9,4)$

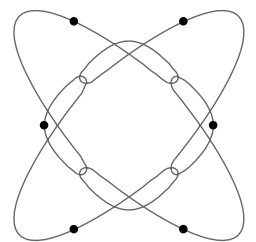

(d) $D(6,4)$

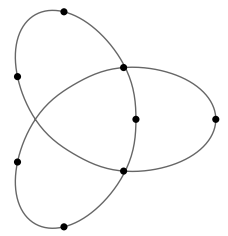

(h) $D(8,3)$

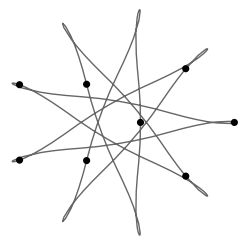

(1) $D(8,9 / 4)$

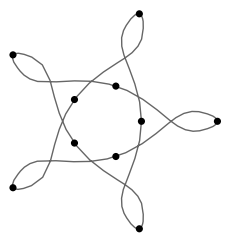

(p) $D(10,5 / 2)$

Figure 2. A selection of choreographies with their symmetry group. See Remark 1.6. These are all from the regular families of symmetry groups; for some examples illustrating the exceptional symmetry groups, see Figure 6 . Where the same label appears for more than one figure, they correspond to different connected components of the corresponding $\operatorname{Fix}\left(G, \Lambda X^{(n)}\right)$. Animations can be viewed on the first author's website [27] and also as supplementary material online at http://dx.doi.org/10.1017/fms.2013.5.

choreographies, and second, his approach is local, and would apply to Hopf bifurcation or Lyapunov centre theorem scenarios: they are the symmetries that can arise for periodic orbits in a linear system. On the other hand, Barutello et al. [2] do give a classification of symmetries for three-body choreographies. 


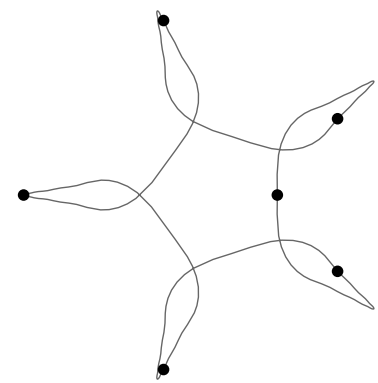

(a) $D(6,5)$

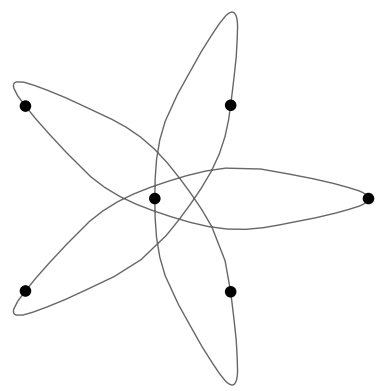

(b) $D(6,5 / 2)$

Figure 3. Comparison of different values of $\ell: D(6,5)$ and $D(6,5 / 2)$ reflect the difference between the symmetries of a pentagon and a pentagram.

Table 1. Comparison of the classification in this paper with that of Barutello et al. [2] for three-particle choreographies (note that in [2] the dihedral group of order $2 m$ is denoted $D_{2 m}$, while here we denote it $\left.D_{m}\right)$.

\begin{tabular}{lllll}
\hline This paper & $D(3, k / \ell)$ & $D^{\prime}(3,2)$ & $D^{\prime}(3,1)$ & $C^{\prime}(3,2)$ \\
Barutello et al. & 'Lagrange' & $D_{6}$ & $D_{3}$ & $C_{6}$
\end{tabular}

However, their classification is simpler than ours (even for $n=3$ ), as they consider the motion in a rotating frame (or modulo rotations), which has the effect of projecting out the rotational part of our symmetry groups, so effectively they consider subgroups of $\mathbb{Z}_{2} \times S_{3} \times \widehat{\mathrm{S}^{1}}$; in particular, all the groups $D(3, k / \ell)$ in this paper are collapsed to the single 'Lagrange type', which is the image of $D(3, \infty / \ell)$ in $\mathbb{Z}_{2} \times S_{3} \times \widehat{\mathrm{S}^{1}}$. See Table 1 . This is similar to viewing the motion on the shape sphere; see for example [30] and references therein.

1.2. Variational problem and topology of loop spaces. The principal motivation for this paper is to apply the results to the variational problem describing the periodic motion of $n$ identical particles in the plane, interacting under a Newtonian potential. However, the Newtonian potential is renowned for its difficulty, as was evident even to Poincaré (in modern terms, because the action functional on the space of collision-free loops is not coercive). The proof of the existence of the figure- 8 solution by Chenciner and Montgomery [8] uses some delicate arguments to show that the minimum of the action functional over the set of loops with the given symmetry cannot occur for a loop with collisions. A different argument for avoiding collisions covering more general 
symmetry classes was given by Ferrario and Terracini [13], under the hypothesis that the symmetry group satisfy what they call the rotating circle condition (see Section 2.6 below).

Given an action of a (Lie) group $\Gamma$ on a manifold $X$, there is a natural action of $\Gamma \times \widehat{\mathrm{S}^{1}}$ on the loop space $\Lambda X$. This action has been used a great deal in bifurcation theory, in particular for the Hopf bifurcation by Golubitsky and Stewart [16] and for the Hamiltonian Lyapunov centre theorem [28]. However, it seems it has not been used as extensively, or as systematically, in variational problems. The second half of this paper goes a little way to address this.

Typically, one is looking for periodic solutions of a differential equation which can be expressed as a variational problem. This includes the existence problem of closed geodesics, as well as periodic orbits for $n$-body problems and more general Lagrangian mechanical systems. Let $\mathcal{A}: \Lambda X \rightarrow \mathbb{R}$ be the 'action functional', whose critical points correspond to periodic solutions of a given fixed period, which we take to be 1 , and assume that it is invariant under the action of $\Gamma \times \widehat{\mathrm{S}}^{1}$. (This invariance occurs for example if the Lagrangian is invariant under the action of $\Gamma$ on $X$, or, for the geodesic problem, if the metric is invariant under the $\Gamma$ action). For each subgroup $G<\Gamma \times \widehat{\mathrm{S}}^{1}$, denote by $\mathcal{A}^{G}$ the restriction of $\mathcal{A}$ to $\operatorname{Fix}(G, \Lambda X)$. By Palais' principle of symmetric criticality [32], critical points of $\mathcal{A}^{G}$ coincide with critical points of $\mathcal{A}$ lying in $\operatorname{Fix}(G, \Lambda X)$, that is, to periodic solutions with spatio-temporal symmetry $G$.

If the functional $\mathcal{A}^{G}$ is coercive, then it is guaranteed to achieve a minimum, and indeed a minimum on each connected component of $\operatorname{Fix}(G, \Lambda X)$. Coercive means that, for every sequence that has no point of accumulation (in the weak topology), the functional tends to infinity, and it is a standard argument in variational calculus that, provided the functional is lower semicontinuous and coercive, then it achieves its minimum; see for example the book of Jost and Li-Jost [23].

For the geodesic problem on a compact Riemannian manifold $X$, the action functional, equal to the length of a loop, satisfies the Palais-Smale condition and is coercive, and the critical points are the closed geodesics [25]. The topological techniques of this paper can be used to prove the existence of symmetric geodesics, namely those satisfying $u(t+\theta)=g \cdot u(t)$, for each $(g, \theta) \in G<$ $\Gamma \times \mathrm{S}^{1}$. In particular, a symmetric geodesic is one that, as a curve in $X$, is invariant under those transformations of $X$ contained in the projection of $G$ to $\Gamma$.

For planar $n$-body problems, the space $X$ is $X^{(n)}$ introduced above, which is not compact, nor even complete because of collisions, and separate arguments are required to deal with the two problems.

Its completion $\overline{X^{(n)}} \simeq \mathbb{C}^{n-1}$ is not compact and the action functional is not coercive, as loops can move to infinity without the action increasing. However, 
imposing restrictions on the types of loops considered can ensure coercivity of $\mathcal{A}$, and there are two types of restriction considered in the literature: topological and symmetry based. The topological constraints were introduced by Gordon [20] using the notion of tied loops. The symmetry approach was used in various ways by different authors and culminated in a beautifully simple result of Ferrario and Terracini [13], who showed that the restriction $\mathcal{A}^{G}$ of $\mathcal{A}$ to the subspace of loops in $\overline{X^{(n)}}$ with symmetry $G$ is coercive if and only if $G<\Gamma \times \widehat{\mathrm{S}^{1}}$ is such that

$$
\operatorname{Fix}\left(G, \overline{X^{(n)}}\right)=\{0\} .
$$

This holds for a wide class of action functions $\mathcal{A}$, including the one derived from the Newtonian potential. For our purposes, this condition (1.6) holds for the choreography group $\mathfrak{C}_{n}$, and a fortiori for any group containing $\mathfrak{C}_{n}$ (the groups of our classification).

There remains the issue of collisions. For the gravitational $1 / r$ potential, the action functional on $\Lambda X^{(n)}$ fails to be coercive because, as was known to Poincaré, there are trajectories with collisions for which the action is finite. Following Poincaré and others since, one can introduce the notion of a strong force (essentially with potential behaving like $1 / r^{a}$ for $a \geq 2$ near collisions, rather than the Newtonian $1 / r$ ), in which case a simple estimate shows that every loop with collisions has infinite action. This idea was investigated by Gordon [20], where he combines it with his idea of tied loops in $\Lambda X^{(n)}$ to ensure that $\mathcal{A}$ is coercive on these connected components of $\Lambda X^{(n)}$. (See also the very interesting papers of Chenciner [5, 6] describing the insights and contributions of Poincaré.)

This discussion leads to the following essentially well-known result.

THEOREM 1.4. Consider the n-body problem with a strong force potential, and let $G$ be any subgroup of $\Gamma \times{\widehat{\mathrm{S}^{1}}}$ containing $\mathfrak{C}_{n}$. Then in each connected component of $\operatorname{Fix}\left(G, \Lambda X^{(n)}\right)$ there is at least one choreographic periodic orbit of the system.

Proof. The strong force analysis by Gordon [20] and the coercivity result of Ferrario and Terracini [13] mentioned above imply that each connected component of $\operatorname{Fix}\left(G, \Lambda X^{(n)}\right)$ contains a local minimum of the action functional. This minimum is a periodic orbit, and necessarily a choreography, since $G$ contains $\mathfrak{C}_{n}$.

Connected components of the loop space $\Lambda X^{(n)}$ correspond to (conjugacy classes of) pure braids, and Montgomery [29] gives a very nice analysis of which components of $\Lambda X^{(n)}$ are tied in Gordon's sense, in terms of the pure braids and their winding numbers. In the second half of this paper, we show that, for each group $G<\Gamma \times \mathrm{S}^{1}$ (so not including time-reversing symmetries, which will be dealt with in a separate paper), the connected components of Fix $\left(G, \Lambda X^{(n)}\right)$ are in 
one-to-one correspondence with certain conjugacy classes in the full braid group, and more precisely by the $P_{n}$-conjugacy classes in a certain coset in $B_{n} / P_{n}$, where $P_{n}$ is the pure braid group on $n$ strings (or twisted conjugacy classes in the case of $\left.C^{\prime}(n, 2)\right)$. The precise formulation is given in Theorem 5.7. In a sense, this can be seen as extending the work of Montgomery.

For the Newtonian (weak) potential, the action functional is not coercive at collisions, and the notion of tied loops does not apply, as the set of collisions does not obstruct moving from one component of $\Lambda X^{(n)}$ to another. The proof by Chenciner and Montgomery [8] of the existence of the figure-8 solution involves showing that the minimum over all loops with collision is greater than the action of a particular loop with the given symmetry (the class $D^{\prime}(3,2)$ in our notation), and hence the minimum over loops with that symmetry must be realized for a collision-free loop. The important paper of Ferrario and Terracini [13] gives a general perturbation argument, based on a technique of Marchal, showing that for many symmetry classes the minimum of $\mathcal{A}^{G}$ cannot be achieved at a trajectory with collisions. These symmetry classes are those satisfying their rotating circle condition, a property we discuss in Section 2.6.

However, even with the gravitational potential, the lack of coercivity does not of course imply that there is not a minimum on each connected component of $\operatorname{Fix}\left(G, \Lambda X^{(n)}\right)$, and indeed numerics suggest that in many, or perhaps most, examples there are such minima (there is numerical evidence that on some connected components there is no minimum, see [34,35], but this evidence is also only numerical).

In this paper, we make no claim to prove explicitly the existence of new choreographies for the Newtonian $n$-body problem, although we can make the following statement, which is an easy consequence of the results of Ferrario and Terracini.

THEOREM 1.5. Suppose that $n$ is odd. Then for each of the symmetry types $D^{\prime}(n, 1)$ and $C^{\prime}(n, 2)$ there is a collision-free periodic orbit of the Newtonian $n$-body system with that symmetry.

It is possible that the choreographies in question are those where $n$ particles move around a figure- 8 curve-however to our knowledge it has not been shown that these minimize the action for the given symmetry type.

Proof. Ferrario and Terracini [13] prove that for any symmetry satisfying the rotating circle condition there is a collision-free minimum in the set of loops with that symmetry. We show in Proposition 2.9 that the symmetry groups in question do satisfy this property.

Note that the groups $C(n, k / \ell)$ also satisfy the rotating circle condition, but in that case it is known that the circular choreography minimizes the action [3]. 


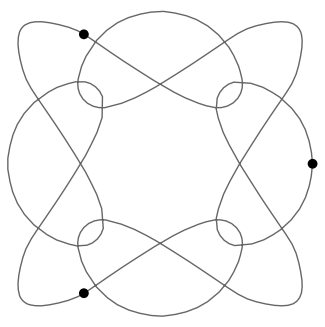

(a) $D(3,4)$ at $t=0$

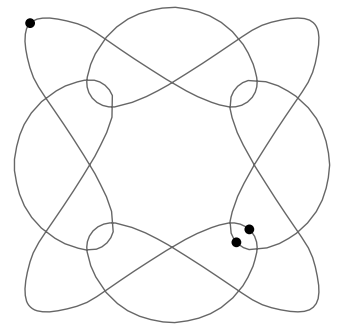

(b) $D(3,4)$ at $t=1 / 24$

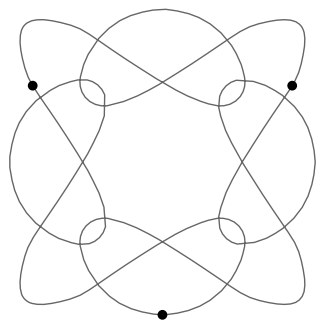

(c) $D(3,4)$ at $t=1 / 12$

Figure 4. A choreography for the three-body problem with fourfold symmetry ('three particles on a Celtic knot'). Note that the particles at $t=1 / 12$ are obtained from those at $t=0$ by rotating by $-\pi / 2$ and relabelling by $\sigma_{1}^{-1}=\left(\begin{array}{lll}1 & 3 & 2\end{array}\right)$. This corresponds to the symmetry element $g=\left(R_{3 \pi / 2}, \sigma_{1}^{2}, 1 / 12\right)$, the generator of $C(3,4)$. Figure (a) demonstrates the element $(\kappa,(23), \overline{0}) \in$ $D(3,4)$, while Figure (b) illustrates $\left(\kappa_{\pi / 4},(13), \overline{1 / 12}\right)$, and Figure (c) illustrates $\left(\kappa_{\pi / 2},(12), \overline{1 / 6}\right)$. See [27] for the animation.

For the three-body problem, many choreographies are known (numerically), almost all of which have just reflectional symmetry (many with one axis, and some with two, such as the figure-8). A new possibility raised here is the symmetry types $D(3, k)$ with $k>2$. A particular case is the choreography of 'three particles on a Celtic knot', depicted in Figure 4, with $D(3,4)$ symmetry. While a motion similar to the figure exists for the strong force, by Theorem 1.4, it would be particularly interesting to know if it exists for the Newtonian attraction.

We have hitherto not been specific about exactly which space of loops we use. For variational calculus one needs the Sobolev space $H^{1}\left(\mathbb{T}, X^{(n)}\right)$ with its usual topology, while for the topological part one uses continuous loops with the compact-open topology. It is proved in [25] that the spaces are homotopic, and the argument can be adapted to show that the homotopy can be chosen to respect the action of $\Gamma \times \widehat{\mathrm{S}^{1}}$. Thus connected components of $\operatorname{Fix}\left(G, C^{0}\left(\mathbb{T}, X^{(n)}\right)\right)$ correspond to those of $\operatorname{Fix}\left(G, H^{1}\left(\mathbb{T}, X^{(n)}\right)\right)$, and the corresponding components are homotopy equivalent.

Organization. The paper is organized as follows. In Section 2, we describe the symmetry groups arising for planar choreographies and some of their properties, and state the classification theorem, Theorem 2.3. The proof of the theorem is the subject of Section 3.

The remainder of the paper investigates the topology of the space of choreographic loops with a given symmetry. Section 4 describes a general approach to the question for the action of any Lie group $\Gamma$ on a manifold $X$. We introduce the notion of 'equivariant fundamental group', which can be used to compute the connected components and their fundamental group of 


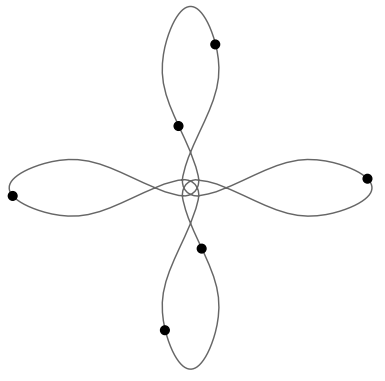

(a) $D(6,4)$

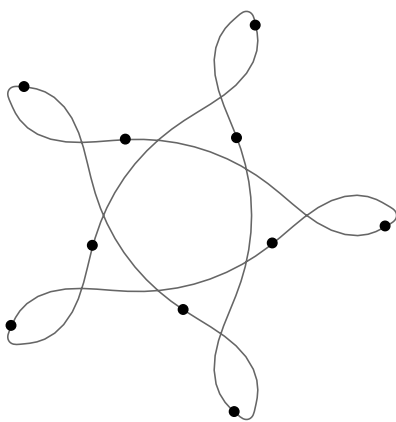

(b) $D(10,5 / 2)$

Figure 5. Two examples of choreographies with nontrivial core: in the first the order of the core is $c=2$, and in the second $c=5$ (generated by rotations through $\pi$ and $2 \pi / 5$, respectively). They are shown for generic values of $t$ illustrating the core symmetry which is valid for all $t$. Compare with special configurations $t=0$ in Figure 2(c) and (p)

the spaces of symmetric loops. These ideas are applied in Section 5 to the question for choreographies. Finally, Section 6 provides a method to describe which components of the space of loops with symmetry $G$ contain loops with symmetry group strictly greater than $G$, an important issue in applying variational techniques. In Sections 4-6, we consider only the action of $\Gamma \times S^{1}$, rather than allowing time-reversing symmetries in $\Gamma \times \widehat{\mathrm{S}}^{1}$. Subgroups with time-reversing symmetries will be considered in a forthcoming paper.

REMARK 1.6. The figures showing choreographies are provided to illustrate the symmetry types of choreographies, and the differences between different connected components of the space of loops with a given symmetry type (a number of examples of this are shown in Figure 2, and compare also Figures 5 and 11). The choreographies are all found using MAPLE or MATLAB by a method of steepest descent to minimize the action for the $n$-body problem with Newtonian potential, although in most cases there is no guarantee that such a solution exists. The method is applied on a space of finite Fourier series with coefficients satisfying conditions corresponding to the symmetry group in question, as described in Section 2.4. Consequently, if there does exist a solution in a particular connected component of the space of loops with a given symmetry type, then it is reasonable to expect the solution to resemble the corresponding figure. Animations of the figures are available on the first author's website [27]; the programming for the animations was created by Dan Gries.

It has been observed by others before that it is not difficult to create numerical examples of choreographies by taking a parameterized curve with several self-intersections and placing on it a large number of particles at regular 
intervals, and then decreasing the action until it is minimized (using a computer programme of course). On the other hand, it appears to be much more of a challenge to produce examples where there are very few particles compared with the degree of symmetry of the curve, such as the examples $D(3,4), D(4,6)$, and $D(5,8)$ illustrated in Figures 2 and 4 . It would be particularly interesting to prove existence results for these examples.

\section{Classification of symmetry types}

2.1. Notation. Here, we introduce some notation for certain subgroups and elements that will be useful throughout the paper. The symbol $n$ always denotes the number of particles, and we assume that $n \geq 3$.

- We denote by $R_{\theta} \in \mathrm{SO}(2)$ the rotation of the plane through an angle $\theta$ and by $\kappa \in \mathrm{O}(2)$ the reflection in the horizontal axis. In complex coordinates, $R_{\theta}$ is multiplication by $e^{i \theta}$ and $\kappa$ is complex conjugation. We occasionally use $\kappa_{\theta}$ to denote reflection in the line at an angle $\theta$ with the horizontal (so $\kappa_{0}=\kappa$ and $\left.R_{\theta} \kappa=\kappa_{\theta / 2}\right)$.

- Let $S_{n}$ denote the symmetric group on $n$ letters, with identity element denoted $e$, and consider two particular subgroups. First, denote by $\sigma_{1} \in S_{n}$ the cycle $\sigma_{1}=(123 \ldots n)$ of order $n$, so $\sigma_{1}(j)=j+1(\bmod n)$, and denote by $\Sigma_{n}<$ $S_{n}$ the cyclic subgroup generated by $\sigma_{1}$. Second, let $s_{1}$ be the order-two permutation which fixes the element 1 and reverses the numerical order: $s_{1}=(2 n)(3(n-1)) \cdots$, so $s_{1}(j)=2-j(\bmod n)$, and denote by $\Sigma_{n}^{+}<S_{n}$ the dihedral group of order $2 n$ generated by $\sigma_{1}$ and $s_{1}$.

- For $k$ coprime to $n$, we denote by $\sigma_{k} \in \Sigma_{n}$ the unique permutation satisfying $\sigma_{k}^{k}=\sigma_{1}$. That is, $\sigma_{k}(j)=j+k^{\prime}$, where $k k^{\prime} \equiv 1(\bmod n)$.

- Recall that $\mathbb{T}$ is the circle $\mathbb{R} / \mathbb{Z}$. For $\theta \in S^{1}$ (the group, also $\mathbb{R} / \mathbb{Z}$ ), denote the transformation $t \mapsto \theta+t$ of $\mathbb{T}$ simply by $\theta$, and the reversing transformation $t \mapsto \theta-t$ by $\bar{\theta}$. Thus 0 denotes the identity, and $\overline{0}$ the reflection about $t=0$. The reflection $\bar{\theta}$ is the reflection that fixes the points $\theta / 2$ and $\theta / 2+1 / 2$.

- The choreography element is $\mathfrak{c}=\left(I, \sigma_{1},-1 / n\right) \in \Gamma \times \mathrm{S}^{1}$, and the choreography subgroup $\mathfrak{C}_{n}$ is the cyclic subgroup of order $n$ generated by $\mathfrak{c}$.

With this notation, the symmetry group of the speed- $\ell$ circular choreography with $n$ particles described in Example 1.3 is generated by

$$
\left(I, \sigma_{1},-\frac{1}{n}\right),\left(R_{2 \pi \ell \theta}, e, \theta\right),\left(\kappa, s_{1}, \overline{0}\right),
$$

with $\theta \in \mathbf{S}^{1}$.

Since the space of choreographies is $\operatorname{Fix}\left(\mathfrak{C}, \Lambda\left(X^{(n)}\right)\right)$, there is a natural action of the normalizer of $\mathfrak{C}_{n}$ in $\Gamma \times{\widehat{\mathrm{S}^{1}}}$ on this space. This normalizer is in fact equal 
to $\mathrm{O}(2) \times \Sigma_{n}^{+} \times \widehat{\mathrm{S}^{1}}$. In the next proposition we show that the isotropy subgroup of any choreography is contained in this group: if collisions were allowed, this would not be the case.

2.2. Basic properties. Associated to any choreography $u$ is its symmetry group $G$, and the projections $\rho, \sigma, \tau$ of $G$ to $\mathrm{SO}(2), S_{n}$, and $\widehat{\mathrm{S}^{1}}$, respectively. We now give a few basic properties of these projections.

PROPOSITION 2.1. Let $u$ be a choreography with symmetry group $G<\Gamma \times \widehat{\mathrm{S}^{1}}$. Then the following hold.

(1) $\operatorname{ker} \tau \cap \operatorname{ker} \rho=\operatorname{ker} \tau \cap \operatorname{ker} \sigma=\mathbf{1}$.

(2) $\operatorname{ker} \tau, \operatorname{ker} \sigma$ and $\operatorname{ker} \rho$ are cyclic groups. Indeed, $\tau(\operatorname{ker} \rho)$ and $\tau(\operatorname{ker} \sigma)$ are subgroups of $\mathrm{S}^{1}$, while $\rho(\operatorname{ker} \tau)$ is a subgroup of $\mathrm{SO}(2)$.

(3) If $\operatorname{ker} \rho \cap \operatorname{ker} \sigma \neq \mathbf{1}$, then the curve is multiply covered. Indeed, if

$$
|\operatorname{ker} \rho \cap \operatorname{ker} \sigma|=\ell>1,
$$

then $u(t / \ell)$ is also a choreography, albeit with a different ordering of the particles.

(4) $\mathfrak{C}_{n}$ is a normal subgroup of $G$, and $\sigma(G)<\Sigma_{n}^{+}$.

A simple example where $\operatorname{ker} \rho \cap \operatorname{ker} \sigma \neq \mathbf{1}$ is the circular choreography with speed $\ell$ for $\ell>1$, see Example 1.3, where $\operatorname{ker} \sigma \cap \operatorname{ker} \rho \simeq \mathbb{Z}_{\ell}$.

Proof. (1) Let $\alpha \in \operatorname{ker} \tau \cap \operatorname{ker} \rho$, in which case $\alpha=(I, \sigma, 0)$ for some $\sigma$. Then $z_{\sigma(i)}(t)=z_{i}(t)$, for all $i, t$. This is not possible without collisions, and so we must have $\sigma=e$.

Now let $\alpha^{\prime} \in \operatorname{ker} \tau \cap \operatorname{ker} \sigma$, so $\alpha^{\prime}=(A, e, 0)$ for some $A \in \mathrm{O}(2)$. Then $A u(t)=$ $u(t)$ for all $t$. If $A$ is a rotational symmetry, then all $z_{i}$ are at the origin for all $t$, leading to collisions, and if $A$ is a reflection, $z_{i}(t) \in \mathbb{R}$ for all $i$, which also implies collisions. Hence $A$ must be trivial.

(2) Assume that $\tau(\operatorname{ker} \rho) \nless \mathrm{S}^{1}$. Then, after possibly reparameterizing time, there exists some $\alpha \in S_{n}$ such that $g=(I, \alpha, \overline{0}) \in \operatorname{ker} \rho$. For this $g, z_{\alpha(j)}(-t)=$ $z_{j}(t) \forall j$, t. If $\alpha(j) \neq j$, then there will be a collision at $t=0$. So we must have $\alpha=e$, and hence $g \in \operatorname{ker} \rho \cap \operatorname{ker} \sigma$; that is, $g=(I, e, \overline{0})$. This implies that $z_{i}(-t)=z_{i}(t)$ for all $i, t$, and so all the particles move in both directions on the same curve. This system has collisions, so is not allowed.

Now assume that $\tau(\operatorname{ker} \sigma)$ is not a subgroup of $S^{1}$, and consider $h=(A, e, \overline{0}) \in$ $\operatorname{ker} \sigma$. Then $h \mathfrak{c} h^{-1} \mathfrak{c}=\left(I, \sigma_{1}^{2}, 0\right)$, which is not possible by part (1) (we assume that $n>2$, so $\sigma_{1}^{2} \neq e$ ). Hence $\tau(\operatorname{ker} \sigma)$ must be a subgroup of $S^{1}$.

Now assume that $\rho(\operatorname{ker} \tau)$ is not a subgroup of $\mathrm{SO}(2)$; that is, there exists some element $k=(\kappa, \sigma, 0) \in \operatorname{ker} \tau$ (up to conjugacy), and we can assume that $\sigma \neq e$ 
by part (1). Since the centre of mass is at the origin and all particles follow the same curve, this curve must intersect $\operatorname{Fix}(\kappa)=\mathbb{R}$. Suppose that $i$ is such that $\sigma(i) \neq i$, and let $t_{0} \in \mathbb{T}$ be such that $z_{1}\left(t_{0}\right) \in \mathbb{R}$. The symmetry element $k$ then guarantees that $z_{\sigma(i)}\left(t_{0}\right)=\kappa z_{i}\left(t_{0}\right)=z_{i}\left(t_{0}\right)-$ a collision! Hence $\rho(\operatorname{ker} \tau)$ must be a subgroup of $\mathrm{SO}(2)$.

It follows that $\operatorname{ker} \sigma$ and $\operatorname{ker} \rho$ must be cyclic, since their images under $\tau$ are cyclic (for $\operatorname{ker} \tau$, its image under $\rho$ ), and their kernels under these maps are trivial.

(3) If $\operatorname{ker} \rho \cap \operatorname{ker} \sigma$ is not trivial, then it must be cyclic, as it is contained in $\operatorname{ker} \rho$. Then there exists $(I, e,(1 / \ell)) \in G$, and we have $u(t+(1 / \ell))=u(t)$ for all $t$, so $u$ is $\ell$-times covered. Moreover, let $\tilde{u}(t)=u(t / \ell)$ for $t \in[0,1]$. This satisfies $\tilde{u}(t+1)=\tilde{u}(t)$, and so it is 1-periodic, and $\tilde{u}$ has choreography symmetry $\left(I, \sigma_{\ell}, 1 / n\right)$. Indeed, set $s=t / l$; then $\tilde{z}_{j}(s+1 / n)=z_{j}(t+(1 / n \ell))=z_{\sigma_{\ell}(j)}(t)=$ $\tilde{z}_{\sigma_{\ell}(j)}(s)$.

(4) Let $\alpha=(A, \pi, a) \in G$, and conjugate $\mathfrak{c}$ by $\alpha$ :

$$
\mathfrak{c}^{\alpha}=\left(I, \pi \sigma_{1} \pi^{-1}, \pm \frac{1}{n}\right) .
$$

The product $\pi \sigma_{1} \pi^{-1}:=\sigma^{\prime}$ will be of the same cycle type as $\sigma_{1}=(1 \ldots n)$, namely a cycle of length $n$. Combine this element $\mathfrak{c}^{\alpha}=\left(I, \sigma^{\prime}, \pm 1 / n\right) \in G$ with $\mathfrak{c}$ or its inverse as needed, depending on the sign of the $\tau$ component, to obtain $\left(I, \sigma^{\prime} \sigma_{1}^{ \pm 1}, 0\right) \in G$. Since the intersection $\operatorname{ker} \tau \cap \operatorname{ker} \rho$ is trivial by part (1), we must have that $\sigma^{\prime} \sigma_{1}^{ \pm 1}=e$ or $\sigma^{\prime}=\sigma_{1}^{\mp 1}$. Then, $\mathfrak{c}^{\alpha}=\left(I, \sigma_{1}^{ \pm 1}, \mp 1 / n\right)$, and hence is in $\mathfrak{C}_{n}$, and so $\alpha$ is in the normalizer of $\mathfrak{C}_{n}$, as required. Finally, since $\sigma_{1}^{\pi}=\sigma_{1}^{ \pm 1}$, the only possibility is that $\pi \in \Sigma_{n}^{+}$(other elements of the normalizer of $\Sigma_{n}^{+}$ conjugate $\sigma_{1}$ to other generators of $\Sigma^{+}$).

COROLlARY 2.2. Let $u$ be a choreography with symmetry $G$. Then either the kernel of $\rho$ is the choreography group $\mathfrak{C}_{n}$, or the choreography is multiply covered.

Proof. First, note that $\mathfrak{C}_{n}<\operatorname{ker} \rho$, by its definition.

Assume that $\operatorname{ker} \rho \neq \mathfrak{C}_{n}$. Then there exists an element $g=(I, \alpha, 1 / r)$ in $G \backslash \mathfrak{C}_{n}$. We know from the proposition that the $\tau$ component of $g$ must not be time reversing, since the spatial component is trivial, and hence there cannot be a reversal of the direction of motion.

Let $m=\operatorname{lcm}(r, n)$ in which case $m>n$. Combination of elements gives $h=(I, \beta,-1 / m)$. We know that $m$ is a multiple of $n$, so set $m=a n$, and then $h^{a}=\left(I, \beta^{a},-1 / n\right)$.

Multiplying by $\mathfrak{c}^{-1}$ gives $\left(I, \sigma_{1}^{-1} \beta^{a}, 0\right)$, which cannot occur by Proposition 2.1, except in the case where $\beta^{a}=\sigma_{1}$, or equivalently $\beta=\sigma_{a}$. In this case, we have $h=\left(I, \sigma_{a},-1 / m\right)$ and $m=a n$, so we obtain an element of $\operatorname{ker} \rho \cap \operatorname{ker} \sigma$, and hence the curve is multiply covered by Proposition 2.1 . 
2.3. Symmetry types. We define some finite subgroups of $\Gamma \times \widehat{\mathrm{S}^{1}}$, which turn out to be all possible finite symmetry types of planar choreographies (Theorem 2.3 below); a symmetry type is a conjugacy class of symmetry groups, or isotropy subgroups. As usual, let $n$ denote the number of particles, and assume that $n \geq 3$, and recall that $\mathfrak{c}=\left(I, \sigma_{1},-1 / n\right)$. There are two regular families and three exceptional subgroups, the latter only for odd values of $n$.

The two regular families are as follows.

$C(n, k / \ell)$ with $k \geq 1, \ell \geq 1$ coprime to $k$; this is the subgroup generated by $\mathfrak{c}$ and $g_{0}=\left(R_{2 \pi \ell / k}, e, 1 / k\right)$. If $\ell=1$, we write this as $C(n, k)$. Clearly $g_{0}$ and $\mathfrak{c}$ commute, so $C(n, k / \ell) \simeq \mathbb{Z}_{n} \times \mathbb{Z}_{k}$, which is cyclic if $n$ and $k$ are coprime. In particular, $C(n, 1)=\mathfrak{C}_{n}$, and if $k>1$ we can restrict to $1 \leq \ell<k / 2$ ( $\ell=0$ would contradict Proposition 2.1; see Remarks 2.4 below).

$D(n, k / \ell)$ with the same conditions on $k, \ell$; this is generated by the two generators $\mathfrak{c}$ and $g_{0}$ of $C(n, k / \ell)$ together with the 'reflection' $\left(\kappa, s_{1}, \overline{0}\right)$ (see Section 2.1 for the notation). The group is isomorphic to $\left(\mathbb{Z}_{n} \times\right.$ $\left.\mathbb{Z}_{k}\right) \rtimes \mathbb{Z}_{2}$, which is an index-2 subgroup of $D_{n} \times D_{k}$ and of order $2 n k$. Indeed,

$$
\begin{aligned}
& D(n, k / \ell) \\
& \quad \simeq\left\{(g, h) \in D_{n} \times D_{k} \mid g, h \text { are both reflections or both rotations }\right\} .
\end{aligned}
$$

If $\ell=1$, we write this as $D(n, k)$. In particular, $D(n, 1)=\mathfrak{C}_{n} \rtimes \mathbb{Z}_{2} \simeq D_{n}$, the dihedral group of order $2 n$.

The three exceptional subgroups, arising only for odd $n$, are as follows.

$C^{\prime}(n, 2)-$ the cyclic group of order $2 n$ generated by $g=\left(\kappa, \sigma_{2},-1 / 2 n\right)$. Notice that $g^{2}=\mathfrak{c}$, and $g^{n}=(\kappa, e, 1 / 2)$.

$D^{\prime}(n, 1)$ - the dihedral group of order $2 n$ generated by $\mathfrak{c}$ and the 'reflection' $\left(R_{\pi}, s_{1}, \overline{0}\right)$.

$D^{\prime}(n, 2)$ - the dihedral group of order $4 n$ generated by $\left(\kappa, \sigma_{2},-1 / 2 n\right)$ and $\left(R_{\pi}, s_{1}, \overline{0}\right)$. This group contains both $C^{\prime}(n, 2)$ and $D^{\prime}(n, 1)$ as index-2 subgroups.

Note that, when dealing with general statements, writing $C(n, k / \ell)$ includes the case $\ell=1$, and clearly $C(n, k / 1)=C(n, k)$, and similarly $D(n, k / 1)=$ $D(n, k)$ (as described in the introduction). See Figures 2-4 for examples of choreographies with symmetry $D(n, k / \ell)$.

We are now ready to state the classification theorem. Recall that the circular choreographies are defined in Example 1.3 and their symmetry is given in (2.1). Consistent with the notation for symmetry groups above, we denote the symmetry group of the circular choreographies by $D(n, \infty / \ell)$. 
THEOREM 2.3. Let $n \geq 3$. The symmetry group of any planar $n$-body choreography is either that of a circular choreography $D(n, \infty / \ell)$ or is conjugate to one of the symmetry groups $C(n, k / \ell), D(n, k / \ell)$, and, if $n$ is odd, $C^{\prime}(n, 2), D^{\prime}(n, 1)$, and $D^{\prime}(n, 2)$.

The proof of this theorem is the subject of Section 3. For the remainder of this section, we make some elementary observations about the symmetry groups in the following remarks, and describe a few consequences of the classification.

REMARKS 2.4. (1) For the groups $C(n, k / \ell)$ and $D(n, k / \ell)$, it is useful to note that the cyclic groups $\operatorname{ker} \rho, \operatorname{ker} \sigma$ and $\operatorname{ker} \tau$ (see Proposition 2.1) are generated as follows:

- $\operatorname{ker} \rho=\mathfrak{C}_{n}$ by $\mathfrak{c}=\left(I, \sigma_{1},-1 / n\right)$;

- $\operatorname{ker} \sigma$ by $g_{0}=\left(R_{2 \pi \ell / k}, e, 1 / k\right)$; and

- $\operatorname{ker} \tau$ (the core), which is cyclic of order $c:=(n, k)$, by

$$
g_{0}^{k / c} c^{n / c}=\left(R_{2 \pi \ell / c}, \sigma_{1}^{n / c}, 0\right) .
$$

- It is also useful to note that if $c=(n, k)=1$ then $\tau$ is an isomorphism so that $C(n, k / \ell)$ is cyclic of order $n k$ generated by

$$
g_{0}^{a} \mathfrak{c}^{b}=\left(R_{2 \pi a \ell / k}, \sigma_{1}^{b}, \frac{1}{n k}\right),
$$

where $a, b$ are such that $a n-b k=1$. More generally, if $(n, k)=c$, then $\tau(G)$ is generated by $c / n k$, and one has

$$
g_{0}^{a} c^{b}=\left(R_{2 \pi a \ell / k}, \sigma_{1}^{b}, \frac{c}{n k}\right),
$$

where now $a n-b k=c$, but $G$ itself is not cyclic.

(2) It was stated in the definition of $C(n, k / \ell)$ that $\ell$ is coprime to $k$. If $(k, \ell)>$ 1 , then the element $(I, e, 1 /(k, \ell))$ is in $G$, contradicting Proposition 2.1. Moreover, the group $C(n, k / \ell)$ is conjugate to $C(n, k /(k-\ell)$ ) (by the element $(\kappa, e, 0))$, and this, together with the coprimality, allows us to restrict attention to $\ell=1$ for $k \leq 4$ and $1 \leq \ell<k / 2$ if $k \geq 5$. The same restrictions also apply to $D(n, k / \ell)$.

(3) If $n$ is even, the analogues of the exceptional subgroups would involve collisions, and so do not arise.

(4) The subgroups denoted $C$ are all nonreversing symmetry groups, while those denoted with $D$ are reversing (that is, of cyclic and dihedral type, respectively, in the language of [13]). 
(5) We will see that all these symmetry types are of interest for choreographies in the $n$-body problem (at least with a strong force interaction-see Theorem 1.4), except those where $n$ divides $k$ (see Example 5.11).

(6) In the two regular families, an element preserves orientation in $\mathbb{T}$ if and only if it preserves orientation in $\mathbb{R}^{2}$, while for the exceptional groups there are elements $g$ with $\tau(g) \in \mathrm{S}^{1}$ but $\rho(g) \notin \mathrm{SO}(2)$, or conversely with $\rho(g) \in \mathrm{SO}(2)$ but $\tau(g) \notin \mathrm{S}^{1}$.

(7) For any choreography with symmetry type $D^{\prime}(n, 1)$ or $D^{\prime}(n, 2)$, the particles pass through the origin. This is because the presence of the time-reversing element $\left(R_{\pi}, s_{1}, \overline{0}\right) \in G$ implies that $z_{1}(0)=R_{\pi} z_{1}(0)$, so that $z_{1}(0)=0$. It then follows that each of the other particles also passes through 0 .

(8) Choreographies of type $C^{\prime}(n, 2)$ may or may not pass through the origin, but if one does not then its winding number around the origin is 0 . This is because the symmetry $\left(\kappa, \sigma_{2}, 1 / 2 n\right)$ reverses the orientation of the plane, but preserves the orientation of the curve, so takes the winding number to its opposite. This is illustrated in Figure 6(a,b), where the origin (the barycentre) is clearly to the left of the symmetric crossing point.

Proposition 2.5. Choreographies with symmetry type $C^{\prime}(n, 2), D^{\prime}(n, 1)$, or $D^{\prime}(n, 2)$ have zero angular momentum.

Proof. The symmetries we consider each have a well-defined effect on angular momentum. The rotations SO(2), the permutations $S_{n}$, and the time translations $S^{1}$ preserve the angular momentum, while the reflections in $O(2)$ and the time-reversing elements of $\widehat{S^{1}}$ change the angular momentum to its opposite. It is clear that any choreography whose symmetry group contains an element which changes the sign of the angular momentum must have angular momentum equal to zero. This is the case for all the exceptional symmetry groups, as $\left(\kappa, \sigma_{2},-1 / 2 n\right)$ and $\left(R_{\pi}, s_{1}, \overline{0}\right)$ reverse the angular momentum (as do all conjugate elements). It is not the case for the regular families.

2.4. Fourier series. Write $z(t)$ for the parameterized curve defining the choreography $u(t)$, with $t \in \mathbb{T}=\mathbb{R} / \mathbb{Z}$. So

$$
z_{j}(t)=z(t+(j-1) / n), \quad j=1, \ldots, n .
$$

Using complex coefficients, we can write $z$ as a Fourier series,

$$
z(t)=\sum_{r \in \mathbb{Z}} \zeta_{r} \exp (2 \pi i r t)
$$

The fact that the centre of mass is at the origin translates into the following constraint on the coefficients, as observed by Simó [35]. 


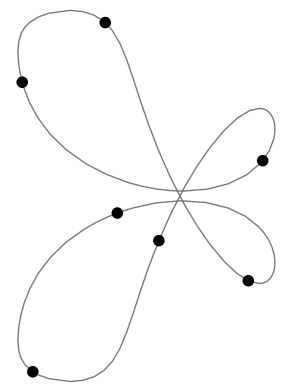

(a) $C^{\prime}(7,2)$ at $t=0$

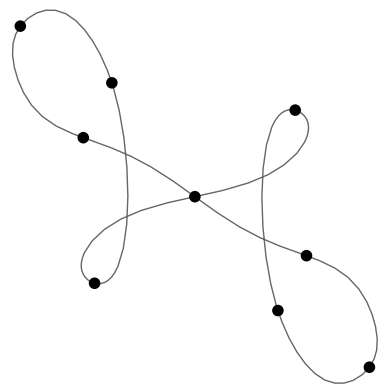

(c) $D^{\prime}(9,1)($ at $t=0)$

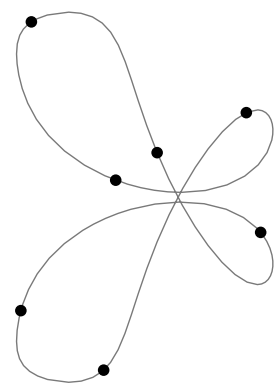

(b) $C^{\prime}(7,2)$ at $t=1 / 14$

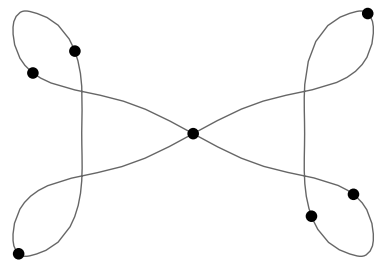

(d) $D^{\prime}(7,2)($ at $t=0)$

Figure 6. Exceptional symmetry groups. (a,b) A choreography for the seven-body problem, with symmetry $C^{\prime}(7,2)$, demonstrating the symmetry generator $\left(\kappa, \sigma_{2}, 1 / 14\right)$, where here $\sigma_{2}=$ (1 526374 ). (c) A choreography with $D^{\prime}(9,1)$ symmetry: rotation by $\pi$ is combined with a time reversal. (d) A choreography with $D^{\prime}(7,2)$ symmetry which possesses both the other symmetries: a rotation by $\pi$ combined with time-reversal as well as a reflection which is not time reversing; another with this symmetry, in a different connected component, would be the figure- 8 curve with seven particles. See [27] for animations.

LEMMA 2.6. If $u$ is a choreography with $n$ particles and $r$ is a multiple of $n$, then $\zeta_{r}=0$ in $(2.5)$.

Proof. Let $z(t)=\sum_{r} \zeta_{r} \exp (2 \pi i r t)$ and $z_{j}(t)=z(t+(j-1) / n)$. The centre of mass (as a function of time) is

$$
\begin{aligned}
0 & =\frac{1}{n} \sum_{r} \zeta_{r}\left[\sum_{j=1}^{n} \exp (2 \pi i r(t+j / n))\right] \\
& =\frac{1}{n} \sum_{r} \exp (2 \pi i r t)\left[\zeta_{r} \sum_{j=1}^{n} \exp (2 \pi i r j / n)\right] .
\end{aligned}
$$


Table 2. Conditions on Fourier coefficients in (2.5) for each symmetry type.

\begin{tabular}{ll}
\hline Symmetry & Conditions on Fourier coefficients \\
$C(n, k / \ell)$ & $\zeta_{r}=0 \forall r \neq \ell(\bmod k)$ \\
$D(n, k / \ell)$ & as $C(n, k / \ell)$, with $\zeta_{r} \in \mathbb{R}$ \\
$C^{\prime}(n, 2)$ & $\zeta_{-r}=(-1)^{r} \bar{\zeta}_{r}, \forall r$ \\
$D^{\prime}(n, 1)$ & $\zeta_{-r}=-\zeta_{r} \forall r$ \\
$D^{\prime}(n, 2)$ & $\zeta_{-r}=-\zeta_{r}, \forall r$ with $\begin{cases}\zeta_{r} \in \mathbb{R} & \text { if } r \text { is even } \\
\zeta_{r} \in \mathrm{i} \mathbb{R} & \text { if } r \text { is odd }\end{cases}$
\end{tabular}

This is satisfied if and only if

$$
\zeta_{r} \sum_{j=1}^{n} \exp (2 \pi i r j / n)=0 \quad(\forall r),
$$

and the result then follows since the sum over $j$ vanishes if $n$ is not a divisor of $r$; otherwise it is equal to $n$.

If the choreography only has choreographic symmetry $\mathfrak{C}_{n}=C(n, 1)$ (as in Figure 1(d)), then there is no further restriction on the Fourier series of the underlying curve. If there is just one reflection giving a time-reversing symmetry, then $z(-t)=\overline{z(t)}$, and this translates into the condition $\zeta_{n} \in \mathbb{R}$, which in practice means that $x(t)$ has a cosine expansion and $y(t)$ a sine expansion (where $z=$ $x+i y)$.

PROPOSITION 2.7. The symmetry of a choreography $u$ translates into the conditions on the Fourier coefficients shown in Table 2.

Proof. This is a simple calculation for each group.

2.5. Isotropy subgroup lattice. For two subgroups $G, H$ of $\Gamma \times \widehat{\mathrm{S}}^{1}$, we write $H \prec G$ to mean that $H$ is subconjugate to $G$ - that is, $H$ is conjugate to a subgroup of $G$. It is a transitive relation.

PROPOSITION 2.8.

The subgroups listed above satisfy the following subconjugacy relations (recall that $C(n, k / 1)=C(n, k)$ and $D(n, k / 1)=D(n, k))$.

(1) $C(n, k / \ell) \prec D(n, k / \ell)$.

(2) $C(n, k / \ell) \prec C\left(n, k^{\prime} / \ell^{\prime}\right)$ if and only if $k \mid k^{\prime}$ and $\ell \equiv \pm \ell^{\prime}(\bmod k)$.

(3) $D(n, k / \ell) \prec D\left(n, k^{\prime} / \ell^{\prime}\right)$ if and only if $k \mid k^{\prime}$ and $\ell \equiv \pm \ell^{\prime}(\bmod k)$.

(4) $C^{\prime}(n, 2) \prec D^{\prime}(n, 2)$ and $D^{\prime}(n, 1) \prec D^{\prime}(n, 2)$.

(5) $C(n, 1) \prec C^{\prime}(n, 2)$ and $C(n, 1) \prec D^{\prime}(n, 1)$.

These are illustrated in Figure 7. Note that (2) and (3) hold with $k^{\prime}=\infty$. 


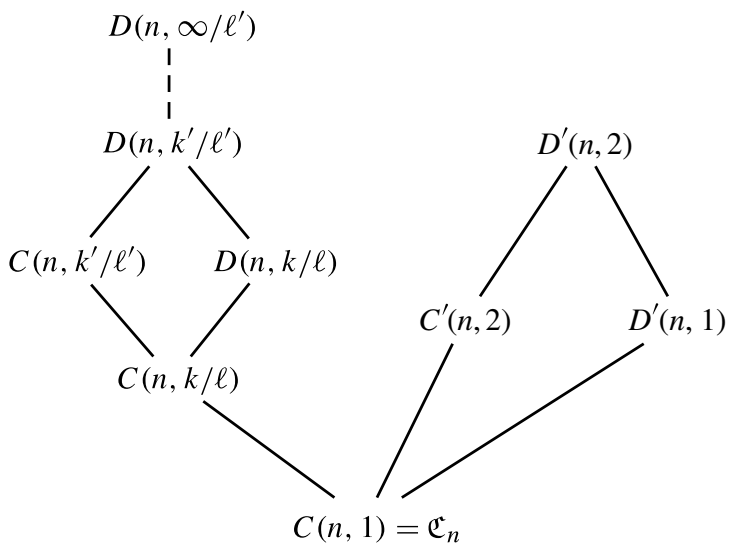

Figure 7. Lattice of isotropy subgroups, with $k \mid k^{\prime}$ and $\ell^{\prime} \equiv \pm \ell(\bmod k)$.

An example illustrating part (2) is that $C(4,5) \prec C\left(4,10 / \ell^{\prime}\right)$ for $\ell^{\prime}=1$ and 4 , while $C(4,5 / 2) \prec C\left(4,10 / \ell^{\prime}\right)$ only for $\ell^{\prime}=3$, as $\ell^{\prime}=2$ is not coprime to $k^{\prime}=10$. Part (3) is similar.

Proof. These all follow from the definitions of the groups (also from the Fourier series representations above). That there are no other subconjugacies is a simple case-by-case analysis, using for example that $G=C(n, k / \ell) \prec G^{\prime}=C\left(n, k^{\prime} / \ell^{\prime}\right)$ requires that $|G|$ divides $\left|G^{\prime}\right|$, and so $k \mid k^{\prime}$. Moreover, the generator of $C^{\prime}(n, 2)$ is not conjugate to any element of any $C(n, k / \ell)$, or indeed of any element of $D(n, \infty / \ell)$.

The importance of this lattice of isotropy subgroups for choreographies lies in the fact that $H \prec G$ is a necessary condition for there to be a sequence of choreographies with symmetry $H$ converging to a choreography with symmetry $G$. For example, for $D(n, \infty / \ell) \prec D(n, k / \ell)$, the choreography determined by

$$
z_{1}(t)=e^{2 \pi i \ell t}(1+\varepsilon \cos (2 \pi k i t)),
$$

which has symmetry $D(n, k / \ell)$, converges as $\varepsilon \rightarrow 0$ to the circular choreography given in Example 1.3, which has symmetry $D(n, \infty / \ell)$. Note also that for $\varepsilon \neq 0$ the particles follow each other in numerical order with time lag $1 / n$, while for $\varepsilon=0$ and $\ell>1$ particle $j$ follows particle $j+m$, where $m \ell \equiv 1(\bmod n)$, but now with time lag $1 / \ell n$.

2.6. Rotating circle condition. In their very interesting paper [13], Ferrario and Terracini introduce the rotating circle condition, and show by a clever perturbation argument that if the action of $G$ satisfies this condition then a collision path in the loop space cannot be a local minimum of the restriction 
of the Newtonian action functional to the set of loops with symmetry $G$. This condition is defined for choreographies in $\mathbb{R}^{d}$, and in $\mathbb{R}^{2}$ it reduces to the following.

A symmetry group $G$ satisfies the rotating circle condition if, for each $t \in$ $[0,1]$, one has

(1) $\rho\left(G_{t}\right)<\mathrm{SO}(2)$; and

(2) $\rho\left(G_{t, i}\right)=\mathbf{1}$, for at least $n-1$ of the indices $i$.

Here, $G_{t}$ is the subgroup of $G$ that fixes $t$ (under $\tau$ ), and $G_{t, i}$ is the subgroup of $G_{t}$ that fixes the index $i$ (under $\sigma$ ). Of course 1 is the trivial group.

Proposition 2.9. The groups $C(n, k / \ell), C^{\prime}(n, 2)$, and $D^{\prime}(n, 1)$ all satisfy the rotating circle condition, while the remaining groups $D(n, k / \ell)$ and $D^{\prime}(n, 2)$ do not.

Proof. For $G=C^{\prime}(n, 2)$, this is immediate, as $G_{t}$ is trivial for all $t$. The same is true of $C(n, k / \ell)$ when $n, k$ are coprime. More generally, for $G=C(n, k / \ell)$, $G_{t}$ is the core generated by $g_{c}=\left(R_{2 \pi \ell / c}, \sigma_{1}^{n / c}, 0\right)$, where $c$ is the order of the core $(\operatorname{ker} \tau)$ - see Remarks 2.4. The first condition is clearly met, as $\rho\left(g_{c}\right)=$ $R_{2 \pi \ell / c} \in \mathrm{SO}(2)$, while the second follows from the fact that $\operatorname{ker} \tau \cap \operatorname{ker} \sigma$ is trivial (Proposition 2.1). For $D^{\prime}(n, 1)$, we have that $G_{t}$ is of order at most two, with $G_{0}$ generated by $g_{0}=\left(R_{\pi}, s_{1}, \overline{0}\right)$. Here $\rho\left(g_{0}\right) \in \mathrm{SO}(2)$, and $\sigma\left(g_{0}\right)=s_{1}$, which fixes precisely one index (namely, 1 - recall that $n$ is odd for $D^{\prime}(n, 1)$ ).

For the two remaining types, we have elements $\left(-\kappa, s_{1} \sigma_{2}, \overline{1 / 2 n}\right) \in D^{\prime}(n, 2)$ and $\left(\kappa, s_{1}, \overline{0}\right) \in D(n, k / \ell)$, both of which violate the rotating circle condition (here $-\kappa=R_{\pi} \kappa$ is the reflection in the vertical axis).

\section{Proof of the classification theorem}

In this section we prove Theorem 2.3, and we rely extensively on the notation introduced in Section 2.1. Let $u$ be a choreography of period 1, with finite symmetry $G$. We also assume that $u$ does not have period less than 1 (if it has minimal period $T<1$ then replace $u(t)$ by $u(t / T)$, which then has period 1 , and if necessary relabel the particles). By Proposition 2.1(4), $G<\mathrm{O}(2) \times \Sigma_{n}^{+} \times \widehat{\mathrm{S}^{1}}$, and $\mathfrak{C}_{n} \triangleleft G$. The proof is in two halves: first, we assume that the symmetry group has trivial core (so $\tau$ is injective), and then we reduce the general case to the first, by considering the quotient by a free group action. As usual, $\tau: G \rightarrow \widehat{\mathrm{S}^{1}}$ is the projection.

3.1. Trivial core. Recall that the core of the symmetry group is $\operatorname{ker} \tau$. So first we assume that $G$ is such that $\tau$ is injective. This means that $G \simeq \tau(G)<\widehat{\mathrm{S}^{1}}$, so that $G$ is isomorphic to a cyclic or a dihedral group. In O $(2)$ and $\widehat{\mathrm{S}^{1}}$, there are two 
nonconjugate of subgroup of order two: the first is the order-two subgroup of $\mathrm{SO}(2)$ or $\mathrm{S}^{1}$, which we denote $C_{2}$ and call cyclic, while the second is generated by a 'reflection' in $\mathrm{O}(2)$ or $\widehat{\mathrm{S}^{1}}$, which we refer to as dihedral, and denote $D_{1}$.

3.1.1. Nonreversing symmetry groups. We are assuming that $\tau(G) \subset \mathrm{S}^{1}$. Since $\tau$ is injective, it follows that $G$ is isomorphic to a subgroup of $\mathrm{S}^{1}$, so is a cyclic group, and since it contains $\mathfrak{C}_{n}$ its order is a multiple of $n$. Suppose then that $G \simeq \mathbb{Z}_{k n}$, where $k$ is a positive integer.

Let $g_{0}$ be the element of $G \simeq \mathbb{Z}_{k n}$ with $\tau\left(g_{0}\right)=1 / n k$ : it is a generator of $G$. Then

$$
g_{0}=\left(\rho\left(g_{0}\right), \sigma\left(g_{0}\right), 1 / n k\right)
$$

for some $\sigma\left(g_{0}\right) \in \Sigma_{n}^{+}$and $\rho\left(g_{0}\right) \in \mathrm{O}(2)$. Consider $g_{0}^{k}$. Now $g_{0}^{k}=$ $\left(\rho\left(g_{0}\right)^{k}, \sigma\left(g_{0}\right)^{k}, 1 / n\right)$, and since $\tau$ is injective this must be equal to $\mathfrak{c}$. It follows that $\rho\left(g_{0}\right)^{k}=I$ and $\sigma\left(g_{0}\right)^{k}=\sigma_{1}$.

The equation $\sigma\left(g_{0}\right)^{k}=\sigma_{1}$ has a solution if and only if $(n, k)=1$. In that case, the solution is unique and is, by definition (see Section 2.1), $\sigma\left(g_{0}\right)=\sigma_{k}$.

Now consider $\rho\left(g_{0}\right)$. Since $\operatorname{ker} \rho=\mathfrak{C}_{n}$ (Corollary 2.2), we have a short exact sequence,

$$
\mathbf{1} \rightarrow \mathfrak{C}_{n} \longrightarrow \mathbb{Z}_{k n} \stackrel{\rho}{\longrightarrow} \mathbb{Z}_{k} \rightarrow \mathbf{1},
$$

where $\mathbb{Z}_{k}<\mathrm{O}(2)$.

- If $k=1$, then $\rho\left(g_{0}\right)=I$ so $G=\mathfrak{C}_{n}=C(n, 1)$.

- If $k>2$, then we have that $\mathbb{Z}_{k}<\mathrm{O}(2)$ is generated by $R_{2 \pi / k}$. The element $\rho\left(g_{0}\right)$ generates $\mathbb{Z}_{k}$, and so must be equal to $R_{2 \pi \ell / k}$ for some $\ell$ coprime to $k$. In this case, we have $G=C(n, k / \ell)$ with $k$ coprime to both $n$ and $\ell$.

- In the case where $k=2$, we have two possibilities. Either $\mathbb{Z}_{k}$ is $C_{2}$, generated by $R_{\pi}$, in which case we have $\rho\left(g_{0}\right)=R_{\pi}$ and $G=C(n, 2)$, or it is dihedral $D_{1}$, generated by $\kappa$ (or a conjugate), in which case $\rho\left(g_{0}\right)=\kappa$, and we have $G=C^{\prime}(n, 2)$.

3.1.2. Reversing symmetry groups. This is a bit more involved. Since we are assuming that the core is trivial, $G \simeq \tau(G)$, and $G$ is therefore a dihedral group. Since $\tau(G)$ contains $(1 / n) \in \mathrm{S}^{1}$, it must be $D_{k n}$ for some $k$. Let $G_{0}=\tau^{-1}\left(\mathbb{Z}_{n k}\right)$ be the index-2 subgroup of $G$ consisting of the nonreversing elements. Let $r \in G$ be an order-two element satisfying $r g=g^{-1} r$ for $g \in G_{0}$, so $r$ together with $G_{0}$ generates $G$. Up to conjugacy by $\mathrm{S}^{1}$, we can suppose that $\tau(r)=\overline{0}$. Furthermore, since $r$ is of order two, it follows that $\rho(r)$ and $\sigma(r)$ are of order one or two.

For $\sigma(r)$, the dihedral condition implies that $r$ anticommutes with $\mathfrak{c}$ and so, in particular,

$$
\sigma(r) \sigma_{1} \sigma(r)^{-1}=\sigma_{1}^{-1}
$$


As $\sigma_{1}$ generates an Abelian group of order $n>2, \sigma(r)$ cannot be in this group. Hence it must be a reflection on the points. Thus, for odd $n, \sigma(r) \in \Sigma_{n}^{+}$must be conjugate to $s_{1}$, which fixes one particle (namely, $z_{1}$ ), while, for even $n$, there are two possibilities:

$$
\sigma(r)= \begin{cases}s_{12} & \text { fixing no particles } \\ s_{1} & \text { fixing } z_{1} \text { and } z_{1+n / 2}\end{cases}
$$

but with $\sigma_{1}$ both generate the same dihedral group $\Sigma_{n}^{+}$.

For the $\rho$ component, since $\rho(r)$ is an element of order at most two, we must have $\rho(r)=I, R_{\pi}$ or a reflection, and this is to be combined with $G_{0}=C(n, k / \ell)$ or, if $n$ is odd, $C^{\prime}(n, 2)$, from the first—nonreversing - part of the proof. However, $\rho(r)=I$ would give an element of $\operatorname{ker} \rho$ whose temporal component is not in $\mathrm{S}^{1}$, contradicting Proposition 2.1(2). There remains to consider $\rho(r)=R_{\pi}$ or a reflection.

The dihedral condition on $r$ gives

$$
\begin{cases}\rho(r) R_{2 \pi \ell / k} \rho(r)^{-1}=R_{-2 \pi \ell / k} & \text { if } G_{0}=C(n, k / \ell) \\ \rho(r) \kappa \rho(r)^{-1}=\kappa & \text { if } G_{0}=C^{\prime}(n, 2) .\end{cases}
$$

Consider each case in turn. First, suppose that $G_{0}=C(n, k / \ell)$.

- If $\rho(r)=R_{\pi}$, it would commute with $R_{2 \pi \ell / k}$ in the above, which is only possible if $k \leq 2$ (recall that $(k, \ell)=1$ ). Then $G$ contains the element $g=$ $\left(R_{\pi}, s, \overline{0}\right)$. If $\bar{k}=2$, then combining $g$ with $\left(R_{\pi}, e, 1 / 2\right) \in C(n, 2)$ produces the element $(I, s, \overline{1 / 2})$, which again contradicts Proposition 2.1(2). If on the other hand $k=1$, we adjoin the element $\left(R_{\pi}, e, \overline{0}\right)$ to $C(n, 1)=\mathfrak{C}_{n}$, which gives the group $D(n, 1)$.

- If $\rho(r)=\kappa$, then $G$ contains $(\kappa, s, \overline{0})$, with $s=s_{1}$ or $s_{12}$ as before, in which case $G$ is of type $D(n, k / \ell)$ : note that together with $C(n, k / \ell)$ the element $(\kappa, s, \overline{0})$ with the two possible values of $s$ generates conjugate subgroups of $\Gamma \times \widehat{\mathrm{S}^{1}}$.

Now suppose that $G_{0}=C^{\prime}(n, 2)$, which is generated by $g^{\prime}=\left(\kappa, \sigma_{2},(1 / 2 n)\right)$ with $n$ odd. By (3.1), $\rho(r)$ commutes with $\kappa$, and so is either $R_{\pi}$ or one of the reflections $\kappa$ or $\kappa^{\prime}:=R_{\pi} \kappa$ (reflection in the line orthogonal to Fix $(\kappa)$ ). Moreover, since $n$ is odd, $\sigma(r)=s_{1}$ (up to conjugacy, or relabelling the particles).

- If $\rho(r)=R_{\pi}$, then $r=\left(R_{\pi}, s_{1}, \overline{0}\right)$, so giving the group $G=D^{\prime}(n, 2)$.

- If $\rho(r)=\kappa$, then $r g^{\prime} \in \operatorname{ker} \rho$ but $\tau\left(r g^{\prime}\right) \notin \mathrm{S}^{1}$, so contradicting Proposition 2.1(2).

- Finally, if $\rho(r)=\kappa^{\prime}$, then $\rho(r \kappa)=\kappa^{\prime} \kappa=R_{\pi}$, and we are in the case $G=$ $D^{\prime}(n, 2)$ again. 


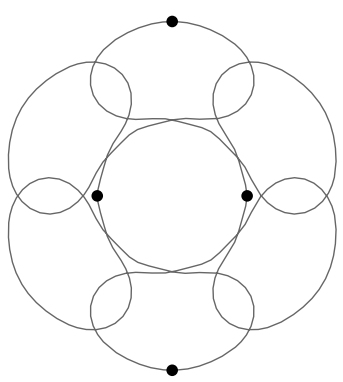

(a) $D(4,6)$ which has core of order 2 , and ....

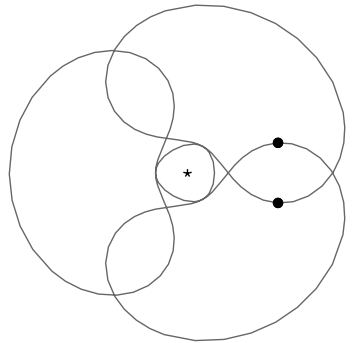

(b) ... its image in $X_{*}^{(2)}$.

Figure 8. A choreography $u$ for the four-body problem with sixfold symmetry, which has core of order two, and its image $\widehat{u}$ under the map $\Psi$.

We have therefore considered every case with a trivial core, and shown that each either gives one of the groups of the classification in Section 2.3 or by contradicting Proposition 2.1 leads to a collision so the trivial core case is complete. We next consider the cases with nontrivial core.

3.2. Nontrivial core. Suppose now that the choreography $u$ has symmetry group $G$ with nontrivial core $K:=\operatorname{ker} \tau$. It follows that $u(t) \in \operatorname{Fix}\left(K, X^{(n)}\right)$ for all $t$. By Proposition 2.1, $K$ is a cyclic group of order $c$, say, and is generated by an element of the form $g_{c}:=\left(R_{2 \pi r / c}, \sigma_{1}^{\hat{n}}, 0\right) \in \Gamma \times \widehat{S^{1}}$ for some $r$ coprime to $c$ (and we may assume that $1 \leq r \leq c / 2$ up to conjugacy). Throughout the paper we put $\widehat{n}=n / c$, and for given $K$ we write $Y=Y_{K}=\operatorname{Fix}\left(K, X^{(n)}\right)$. For the choreography to have core $K$ it follows that, for all $j$,

$$
z_{j+\widehat{n}}=e^{2 \pi i r / c} z_{j} .
$$

We begin with an elementary lemma. Recall that the centre of mass is fixed at the origin.

LEMMA 3.1. Any choreography with nontrivial core does not pass through the origin.

Proof. Let $g_{c}=\left(R_{2 \pi r / c}, \sigma_{1}^{n / c}, 0\right)$ generate the core, and suppose for contradiction that the curve does pass through the origin: say $z_{1}\left(t_{0}\right)=0$. Then $z_{\widehat{n}+1}\left(t_{0}\right)=$ $e^{2 \pi i r / c} 0=0$, resulting in a collision.

The classification of choreographies in $Y$ proceeds by identifying $Y$ as a smooth finite cover of $X_{*}^{(\widehat{n})}$, the configuration space of $\widehat{n}$ distinct particles in the punctured plane $\mathbb{C} \backslash\{0\}$, thereby reducing the classification of choreographies 
with core to those with no core, but now in $X_{*}^{(\widehat{n})}$. Explicitly, the covering is given by

$$
\begin{aligned}
\Psi: \operatorname{Fix}\left(K, X^{(n)}\right) & \longrightarrow X_{*}^{(\widehat{n})} \\
\left(z_{1}, \ldots, z_{n}\right) & \longmapsto\left(z_{1}^{c}, \ldots, z_{\widehat{n}}^{c}\right) .
\end{aligned}
$$

Note that it follows from the lemma that the $z_{j} \neq 0$. Solving (3.3) and using (3.2) shows that $\Psi$ is a smooth covering of degree $c^{n}$.

Let $u(t)=\left(z_{1}(t), \ldots, z_{n}(t)\right.$ be a choreography with core $K$, and define the loop $\widehat{u}$ in $X_{*}^{(\widehat{n})}$ by

$$
\widehat{u}(t)=\left(w_{1}(t), \ldots, w_{\widehat{n}}(t)\right)=\Psi(u(t / c)),
$$

which implies that $w_{j}(t)=z_{j}(t / c)^{c}$. Combining (3.2) with the choreography symmetry of $u$ shows that each $w_{j}$ is of period 1, and the loop $\widehat{u}$ is indeed a choreography. Furthermore, if the core of $u$ is precisely $K$, then the core of $\widehat{u}$ is trivial, so we can apply the classification we have so far obtained (Section 3.1) to $\widehat{u}$.

We can immediately rule out the exceptional groups $D^{\prime}(n, 1)$ and $D^{\prime}(n, 2)$, as they necessarily pass through the origin (which is excluded by the lemma above). We can also rule out $C^{\prime}(n, 2)$, as its winding number is necessarily zero (see Remarks 2.4(8)), while we have the following lemma.

LEMma 3.2. Any choreography $u$ with nontrivial core has nonzero winding number around the origin.

Proof. Let $u(t)=\left(z_{1}(t), \ldots, z_{n}(t)\right)$, and consider the underlying curve $z(t)=$ $z_{1}(t)$ say. The winding number is given by integrating $d z / z$ around the curve. First, integrate $d z / z$ from $t=0$ to $t=1 / c$. This is equivalent to $\ln (z(1 / c)-\ln (z(0))(\bmod 2 \pi i)$, which is $2 \pi i(r / c(\bmod 1)) \neq 0$, using the choreography symmetry and (3.2). The integral from $t=0$ to $t=1$ is therefore $2 \pi i(r(\bmod c)) \neq 0$, and so the winding number itself is equivalent to $r(\bmod c)$, and we are done.

There remain the possibilities of $\widehat{u}$ having symmetry $C(\widehat{n}, \widehat{k} / \widehat{\ell})$ or $D(\widehat{n}, \widehat{k} / \widehat{\ell})$, for some integers $\widehat{k}, \widehat{\ell}$ with $(\widehat{n}, \widehat{k})=(\widehat{k}, \widehat{\ell})=1$. So the question remains, given one of these symmetries for $\widehat{u}$, what are the possible symmetries of $u$ ?

Suppose first that $\widehat{u}$ has symmetry $C(\widehat{n}, \widehat{k} / \widehat{\ell})$, for $\widehat{\ell}$ coprime to $\widehat{k}$. We claim that $u$ has symmetry (conjugate to) $C(n, k / \ell)$ for some $\ell$ necessarily coprime to $k=c \widehat{k}$ and with $\ell= \pm \widehat{\ell}(\bmod \widehat{k})$. Note that since $c \mid n$ and $(\widehat{n}, \widehat{k})=1$ it follows that $(n, k)=c$, consistent with the fact that the core is of order $c$.

Now $C(\widehat{n}, \widehat{k} / \widehat{\ell})$ is generated by the choreography element $\hat{\mathfrak{c}}=\left(I, \widehat{\sigma}_{1},-1 / \widehat{n}\right)$ and $\widehat{g}_{0}=\left(R_{2 \pi \widehat{\ell} \widehat{k}}, e, 1 / \widehat{k}\right)$. Explicitly, for each $j$ and each $t$,

$$
e^{2 \pi \hat{\imath} \widehat{\ell} \widehat{k}} w_{j}\left(t-\frac{1}{\widehat{k}}\right)=w_{j}(t) \text {. }
$$


Taking $c$ th roots and replacing $t$ by $t / c$ gives

$$
e^{2 \pi i \widehat{\ell} / k} z_{j}\left(t-\frac{c}{n k}\right)=e^{2 \pi i h / c} z_{j}(t),
$$

for some integer $h$ (independent of $j$ and $t$ by the choreography symmetry), with $k=c \widehat{k}$. That is, the symmetry group of $u$ contains, in addition to $K$ and $\mathfrak{C}_{n}$, the group generated by

$$
g=\left(R_{2 \pi(\widehat{\ell}-h \widehat{k}) / k}, e,(c / n k)\right) .
$$

This is the symmetry group $C(n, k / \ell)$ with $\ell=\widehat{\ell}+h \widehat{k}$ as required. Note that $r$ in the core is related to $\ell$ by $r \equiv \ell(\bmod c)$.

Finally, suppose that $\widehat{u}$ has symmetry $D(\widehat{n}, \widehat{k} / \widehat{\ell})$. Then it also has symmetry $C(\widehat{n}, \widehat{k} / \widehat{\ell})$, and so $u$ has symmetry $C(n, k / \ell)$ as above. Moreover, $\widehat{u}$ has symmetry $\left(\kappa, s_{1}, \overline{0}\right)$. That is,

$$
\bar{w}_{2-j}(-t)=w_{j}(t) .
$$

Here, the index $2-j$ is modulo $\widehat{n}$. Lifting this to $u$ (taking $c$ th roots and replacing $t$ by $t / c$ as above) gives

$$
\bar{z}_{2-j+f \hat{n}}(-t)=z_{j}(t),
$$

for some $f$. This leads to a subgroup conjugate to $D(n, k / \ell)$, and concludes the proof of Theorem 2.3.

\section{Connected components of spaces of symmetric loops}

Let us fix notation. Throughout the remainder of this paper, $X$ will denote a smooth manifold, and $\Gamma$ will be a Lie group acting smoothly on $X$. (Although we assume $X$ to be a manifold, the results hold more generally.) The loop space $\Lambda X$ on $X$ is the set of all continuous maps $u: \mathbb{T} \rightarrow X$, where we identify the circle $\mathbb{T}$ with $\mathbb{R} / \mathbb{Z}$. All loop spaces are given the compact-open topology. There is then a natural action of $\Gamma \times \mathrm{S}^{1}$ on the loop space $\Lambda X$ given by

$$
((g, \theta) \cdot u)(t)=g \cdot u(t-\theta) .
$$

We will consider the natural action of $\Gamma \times \widehat{S^{1}}$ in a later paper. For the remainder of this paper, we change notation slightly compared to the earlier sections. Instead of writing $G<\Gamma \times \mathrm{S}^{1}$ as hitherto, we now let $G$ be a subgroup of $\Gamma$, and the corresponding subgroup of $\Gamma \times \mathrm{S}^{1}$ is given as the graph of a homomorphism $\tau: G \rightarrow \mathrm{S}^{1}$; we denote this subgroup by $G_{\tau}$.

In this section, we analyse the sets of connected components of the spaces $\operatorname{Fix}\left(G_{\tau}, \Lambda X\right)$ for subgroups $G_{\tau}$ of $\Gamma \times \mathrm{S}^{1}$. In the following section, we apply these results to spaces of choreographies with $C(n, k / \ell)$ and $C^{\prime}(n, 2)$ symmetry. 
In a future paper, we will consider the reversing symmetry groups in $\Gamma \times \widehat{\mathrm{S}^{1}}$, with applications to the remaining symmetry types of choreography.

The topological analysis in this section is based on [26], but expressed in more 'equivariant' terms.

4.1. Symmetric loops. We assume throughout that $X$ is a manifold with an action of a Lie group $\Gamma$, and we let $\tau: G \rightarrow \mathrm{S}^{1}$ be a given homomorphism, and write $K=\operatorname{ker} \tau \triangleleft G$. The graph $G_{\tau}$ of $\tau$ is of course the subgroup $\{(g, \tau(g)) \in$ $\left.\Gamma \times \mathrm{S}^{1} \mid g \in G\right\}$. A loop $u \in \Lambda X$ is said to have symmetry $G_{\tau}$ if $u \in \operatorname{Fix}\left(G_{\tau}, \Lambda X\right)$. This means that $(g, \tau(g)) \cdot u=u$ for all $g \in G$, or $g \cdot u(t)=u(t+\tau(g))$.

The following lemma is easy to check.

LEMMA 4.1. The action of $G_{\tau}$ on $\Lambda X$ restricts to an action of $(G / K)_{\tau}$ on $\Lambda\left(X^{K}\right)$, where $X^{K}=\operatorname{Fix}(K, X)$. Moreover, $\operatorname{Fix}\left(G_{\tau}, \Lambda X\right)=\operatorname{Fix}\left((G / K)_{\tau}, \Lambda\left(X^{K}\right)\right)$.

For $g \in \Gamma$, let

$$
\Lambda^{g} X=\{\gamma:[0,1] \rightarrow X \mid \gamma(1)=g \cdot \gamma(0)\}
$$

be the space of relative loops with phase $g$, or more briefly of g-loops. In particular, $\Lambda^{e} X=\Lambda X$.

For each $g \in \Gamma$ and each positive integer $r$, define a map $\phi_{r}: \Lambda^{g} X \rightarrow \Lambda^{g^{r}} X$ by concatenating $\gamma$ with its image under $g$, and then with its image under $g^{2}$, and so on, up to $g^{r-1}$. That is,

$$
\phi_{r}(\gamma)(t)= \begin{cases}\gamma(r t) & \text { if } t \in[0,1 / r] \\ g \gamma(r t-1) & \text { if } t \in[1 / r, 2 / r] \\ \vdots & \\ g^{r-1} \gamma(r t-r+1) & \text { if } t \in[(r-1) / r, 1] .\end{cases}
$$

Note that indeed $\phi_{r}(\gamma)(0)=\gamma(0)$ and $\phi_{r}(\gamma)(1)=g^{r} \gamma(0)$. Furthermore, if $g^{r}=e$, then $\phi_{r}(\gamma) \in \operatorname{Fix}(H, \Lambda X)$, where $H$ is the cyclic group generated by $(g, 1 / r) \in \Gamma \times \mathrm{S}^{1}$.

Proposition 4.2. Suppose that $\tau(G)$ is of order $r$, in which case $\tau(G)=\mathbb{Z}_{r}<$ $\mathrm{S}^{1}$. Then the map

$$
\phi_{r}: \Lambda^{g} X^{K} \longrightarrow \operatorname{Fix}\left(G_{\tau}, \Lambda X\right),
$$

defines a homeomorphism for any $g \in G$ satisfying $\tau(g)=1 / r$.

Note that if $g, g^{\prime}$ satisfy $\tau(g)=\tau\left(g^{\prime}\right)$ then they differ by an element of $K$, and therefore they act the same way on $X^{K}$, and so $\Lambda^{g} X^{K}=\Lambda^{g^{\prime}} X^{K}$. 
Proof. Let $\gamma \in \Lambda^{g} X^{K}$ and $u=\phi_{r}(\gamma)$. Since $\gamma(t) \in X^{K}$ for all $t$, so is $u(t)$. Since $g^{r} \in K$, it follows that $u$ is indeed a loop. That $u$ has symmetry $G_{\tau}$ is straightforward. Moreover, $\phi_{r}$ is a homeomorphism, as the inverse is simply the restriction $u \mapsto[t \mapsto u(t / r)]$ (with $t \in[0,1]$ ), and both maps are continuous in the compact-open topology.

Definition 4.3. Given a loop $u \in \Lambda X$ with symmetry $G_{\tau}$ as above, we call $\gamma=\phi_{r}^{-1}(u)$ a generator of $u$. Explicitly,

$$
\gamma(t)=u(t / r), \quad t \in[0,1] .
$$

4.2. Equivariant loop space. Let $X$ be a manifold, and let $\Gamma$ be a group acting on $X$. Denote by $\mathcal{P}(X)$ the space of all paths on $X$, that is, continuous maps $[0,1] \rightarrow X$, and by $\mathcal{P}(X, x)$ the space of those paths $\gamma$ for which $\gamma(0)=x$. We follow standard notation, and denote by $\Lambda X$ the space of (free) loops in $X$, that is the set of those $\gamma \in \mathcal{P}(X)$ for which $\gamma(1)=\gamma(0)$, and we let $\Omega(X, x)$ denote the space of loops based at $x$, namely those for which $\gamma(0)=\gamma(1)=x$. All spaces of paths are given the compact-open topology.

We define the equivariant free loop space to be

$$
\Lambda^{\Gamma} X:=\{(\gamma, g) \in \mathcal{P}(X) \times \Gamma \mid \gamma(1)=g \cdot \gamma(0)\} .
$$

Similarly, for $x \in X, \Omega^{\Gamma}(X, x)$ consists of those equivariant loops $(\gamma, g)$ with $\gamma(0)=x$ and $\gamma(1)=g \cdot x$. We will denote by $\beta$ the projection $\mathcal{P}(X) \times \Gamma \rightarrow \Gamma$ given by $\beta(\gamma, g)=g$. We also denote the restrictions of $\beta$ to $\Lambda^{\Gamma} X$ and $\Omega^{\Gamma}(X, x)$ by $\beta$. In the last section we defined, for each $g \in \Gamma$,

$$
\Lambda^{g} X=\{\gamma \in \mathcal{P}(X) \mid \gamma(1)=g \cdot \gamma(0)\}=\beta^{-1}(g) \subset \Lambda^{\Gamma} X .
$$

In the same way that the fundamental group $\pi_{1}(X, x)$ is defined to be $\Omega(X, x) / \sim$, where $\sim$ means homotopy of paths relative to the end points, we define

$$
\pi_{1}^{\Gamma}(X, x)=\Omega^{\Gamma}(X, x) / \sim,
$$

where again $\sim$ is relative to end points, and with $g$ kept fixed. This set $\pi_{1}^{\Gamma}(X, x)$ has a natural group structure given by

$$
(\gamma, g)(\delta, h)=(\gamma *(g \delta), g h),
$$

where $g \delta$ is the image of $\delta$ under the action of $g$ on $X$, and $*$ is the usual concatenation product in homotopy. Note that our notation does not distinguish between homotopy classes and their representatives: one could be more careful and write for example $([\gamma], g)([\delta], h)=([\gamma * g \delta], g h)$. We hope that this will not be a source of confusion. Some details and examples of this group can be found in the second author's thesis [37, Section 2.4]. The group was introduced by Rhodes [33], where he calls it the fundamental group of the transformation 
group, and denotes it $\sigma(X, x, \Gamma)$. The group is independent of base point $x$ up to isomorphism, provided $X$ is path connected, or more generally provided $\Gamma$ acts transitively on the set of connected components of $X$. (Rhodes [33] proves this only for Abelian groups, but it is easily seen to be true in general: indeed the map $(\gamma, g) \mapsto\left(h \gamma, h g h^{-1}\right)$ provides a homeomorphism $\Omega^{\Gamma}(X, x) \rightarrow$ $\Omega^{\Gamma}(X, h x)$ which descends to an isomorphism $\pi_{1}^{\Gamma}(X, x) \rightarrow \pi_{1}^{\Gamma}(X, h x)$.) Some other properties, immediate from the definition, are as follows. We write all groups multiplicatively, so the trivial (homotopy) group is denoted $\mathbf{1}$.

- There is a short exact sequence,

$$
\mathbf{1} \rightarrow \pi_{1}(X, x) \longrightarrow \pi_{1}^{\Gamma}(X, x) \stackrel{\beta}{\longrightarrow} \Gamma \rightarrow \mathbf{1},
$$

where $\beta$ is the natural projection $(\gamma, g) \mapsto g$ as above, and more generally, if $N \triangleleft \Gamma$ (normal subgroup), then

$$
\mathbf{1} \rightarrow \pi_{1}^{N}(X, x) \longrightarrow \pi_{1}^{\Gamma}(X, x) \longrightarrow \Gamma / N \rightarrow \mathbf{1} .
$$

- If $H<\Gamma$, then $\pi_{1}^{H}(X, x)=\beta^{-1}(H)<\pi_{1}^{\Gamma}(X, x)$.

- If $x$ is fixed by $\Gamma$, then $\pi_{1}^{\Gamma}(X, x) \simeq \pi_{1}(X, x) \rtimes \Gamma$, where the action of $\Gamma$ on $\pi_{1}(X, x)$ is the natural one. In particular, if the action of $\Gamma$ on $X$ is trivial, then $\pi_{1}^{\Gamma}(X, x) \simeq \pi_{1}(X, x) \times \Gamma$.

- If $f: X \rightarrow Y$ is an equivariant map, then there is a natural homomorphism $f_{*}: \pi_{1}^{\Gamma}(X, x) \rightarrow \pi_{1}^{\Gamma}(Y, f(x))$. More generally, if $\Gamma<H$ and $f: X \rightarrow Y$ is $\Gamma$-equivariant, then there is a natural homomorphism

$$
f_{*}: \pi_{1}^{\Gamma}(X, x) \longrightarrow \pi_{1}^{H}(Y, f(x)) .
$$

At the level of homotopy, the homeomorphism $\phi_{r}$ of Proposition 4.2 becomes

$$
\left(\phi_{r}(\gamma), g^{r}\right)=(\gamma, g)^{r},
$$

using the group structure in $\pi_{1}^{\Gamma}$.

EXAMPLE 4.4. Let $S^{1}$ act on a manifold $X$. Then there is a homomorphism $\alpha: \pi_{1}\left(\mathrm{~S}^{1}, e\right) \rightarrow \pi_{1}(X, x)$ generated by the homotopy class of the orbit $\mathrm{S}^{1} \cdot x$. It is well known that the image of $\alpha$ lies in the centre of $\pi_{1}(X, x)$. Then we claim that

$$
\pi_{1}^{\mathrm{S}^{1}}(X, x) \simeq \pi_{1}(X, x) \times{ }_{\alpha} \mathbb{R},
$$

where $\times_{\alpha}$ means that we identify $(\gamma, r+n)$ and $(\alpha(n) \gamma, r)$ for $n \in \mathbb{Z} \simeq$ $\pi_{1}\left(\mathrm{~S}^{1}, e\right)$; in other words, we factor $\pi_{1}(X, x) \times \mathbb{R}$ by the normal subgroup generated by $(\alpha(1),-1)$, where the 1 is the generator of $\pi_{1}\left(S^{1}, e\right) \simeq \mathbb{Z}<\mathbb{R}$. The isomorphism is given as follows. Let $\theta \in \mathbb{R}$ and $p \in X$, and denote by $\theta_{p}$ the path

$$
\theta_{p}(t)=(t \theta) \cdot p
$$


which is a path from $p$ to $\theta \cdot p$ following the $S^{1}$-orbit. Then, for $(\gamma, \theta) \in$ $\pi_{1}(X, x) \times \mathbb{R}$, the corresponding element of $\pi_{1}^{\mathrm{S}^{1}}(X, x)$ is $\left(\gamma * \theta_{x}, \theta(\bmod 1)\right)$. This descends to a well-defined homomorphism $\pi_{1}(X, x) \times_{\alpha} \mathbb{R} \rightarrow \pi_{1}^{\mathrm{S}^{1}}(X, x)$, which has inverse

$$
(\gamma, \theta) \mapsto\left[\gamma *(-\theta)_{\theta \cdot x}, \theta\right] .
$$

Note that $(-\theta)_{\theta \cdot x}$ is the reverse path of $\theta_{x}$. (Everything is up to homotopy of course.)

The projection $\beta$ makes $\pi_{1}^{\mathrm{S}^{1}}(X, x)$ into a bundle over $\mathrm{S}^{1}$ with fibre $\pi_{1}(X, x)$ and monodromy $\alpha$. If we restrict the action to the cyclic group $\mathbb{Z}_{k}<\mathrm{S}^{1}$, then the corresponding subgroup of $\pi_{1}(X, x) \times_{\alpha} \mathbb{R}$ is

$$
\pi_{1}^{\mathbb{Z}_{k}}(X, x) \simeq \pi_{1}(X, x) \times_{\alpha} \mathbb{Z}(1 / k),
$$

where $\mathbb{Z}(1 / k)$ is the subgroup of $\mathbb{R}$ consisting of integer multiples of $1 / k$.

A fundamental property of $\pi_{1}^{\Gamma}(X, x)$ is the following. The first part in fact follows from a result of Rhodes [33, Theorem 4], although he proves a more general statement about orbit spaces of not necessarily free actions. The argument in the free case is more straightforward, so we give it here (it appears also in the second author's thesis [37, Proposition 2.4.3]).

Proposition 4.5. If $N$ is finite and acts freely on a manifold $X$, then $\pi_{1}^{N}(X, x) \simeq \pi_{1}(X / N, \bar{x})$; more generally, if $N$ is a finite normal subgroup of $\Gamma$ and acts freely on $X$, then

$$
\pi_{1}^{\Gamma}(X, x) \simeq \pi_{1}^{\Gamma / N}(X / N, \bar{x}) .
$$

Proof. The map $X \rightarrow X / N$ is a covering, and so it enjoys the homotopy lifting property (see, for example, Hatcher [22, Proposition 1.30]). Consider the natural map given by projecting the path:

$$
\Omega^{N}(X, x) \longrightarrow \Omega(X / N, \bar{x}) .
$$

It follows from the homotopy lifting property that this map is a homeomorphism, and that it induces the required isomorphism

$$
\pi_{1}^{N}(X, x) \longrightarrow \pi_{1}\left(X_{N}, \bar{x}\right) .
$$

The more general statement follows from the snake lemma applied to the diagram.

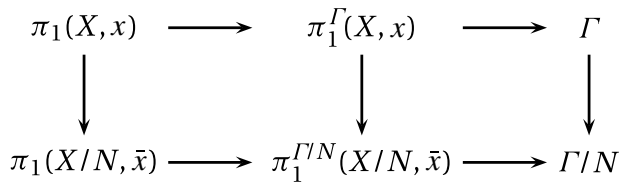


The first column is injective with cokernel $N$, since $\pi_{1}^{N}(X, x) \simeq \pi_{1}(X / N, \bar{x})$, and the final column is surjective with kernel $N$; since the resulting homomorphism $N \rightarrow N$ is an isomorphism, it follows that the middle column is an isomorphism.

A simple consequence of this is that, if $\Gamma$ is a finite group, $\pi_{1}^{\Gamma}(X, x)$ is the equivariant fundamental group of $X$.

COROLlARY 4.6. If $\Gamma$ is a finite group acting on a manifold $X$, then $\pi_{1}^{\Gamma}(X, x)$ is isomorphic to the fundamental group of $X_{\Gamma}:=X \times_{\Gamma} \mathrm{E} \Gamma$.

Here, $\mathrm{E} \Gamma$ is the total space of the universal classifying bundle for $\Gamma$; it is a contractible space on which $\Gamma$ acts freely. The space $X \times_{\Gamma} \mathrm{E} \Gamma=(\mathrm{E} \Gamma \times X) / \Gamma$ is (the Borel construction for) the homotopy orbit space for the $\Gamma$-action on $X$; see for example [21].

Proof. Let $* \in E$ be a base point, and for each $g \in \Gamma$ choose a path $\omega_{g}$ from $*$ to $g \cdot *$ (since $\mathrm{E} \Gamma$ is contractible, $\omega_{g}$ is unique up to homotopy). Consider the injective map $i: \Omega^{\Gamma}(X, x) \rightarrow \Omega^{\Gamma}(X \times \mathrm{E} \Gamma,(x, *))$ defined by $i(\gamma, g)=$ $\left(\left(\gamma, \omega_{g}\right), g\right)$.

We claim that the induced homomorphism

$$
i_{*}: \pi_{1}^{\Gamma}(X, x) \longrightarrow \pi_{1}^{\Gamma}(X \times \mathrm{E} \Gamma,(x, *))
$$

is an isomorphism. That it is injective is clear, for, if $(\gamma, g)$ is in the kernel, then $g=e$, and $\gamma$ is trivial. It is surjective because $\mathrm{E} \Gamma$ is contractible, so any $\Gamma$-loop $\left(\left(\gamma, \delta_{g}\right), g\right)$ is homotopic to $\left(\left(\gamma, \omega_{g}\right), g\right)$, which is in the image of the original map $i$. Therefore

$$
\pi_{1}^{\Gamma}(X, x) \simeq \pi_{1}^{\Gamma}(X \times \mathrm{E} \Gamma,(x, *)) \simeq \pi_{1}\left(X_{\Gamma}, \overline{(x, *)}\right),
$$

where the latter isomorphism follows from the proposition above.

4.3. Connected components. We are interested in the topology of the space $\Lambda^{\Gamma} X$ of equivariant loops, and in particular of each $\Lambda^{g} X=\beta^{-1}(g)$. To this end we adapt the usual argument showing that the connected components of the free loop space $\Lambda X$ correspond to conjugacy classes in the fundamental group $\pi_{1}(X, x)$.

First, we need the topology of the based equivariant loop space $\Omega^{\Gamma}(X, x)$, or rather $\Omega^{g}(X, x)$ for each $g$. This is derived by giving $\Gamma$ the discrete topology and using the fibration $\mathcal{P}(X, x) \times \Gamma \rightarrow X,(\gamma, g) \mapsto g^{-1} \gamma(1)$ with fibre $\Omega^{\Gamma}(X, x)$. Since $\mathcal{P}(X, x)$ is contractible, one finds from the long exact sequence that, for $k \geq 1$,

$$
\pi_{k}\left(\Omega^{\Gamma}(X, x),(\gamma, g)\right) \simeq \pi_{k+1}(X, x)
$$


And by definition $\pi_{0}\left(\Omega^{\Gamma}(X, x),(\gamma, g)\right)=\pi_{1}^{\Gamma}(X, x)$ (as $\Gamma$ has the discrete topology).

Consider now the fibration in the following diagram.

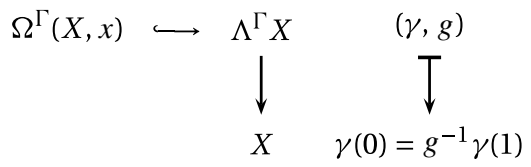

The fibre over the point $x$ is $\Omega^{\Gamma}(X, x)$. The long exact sequence associated to this fibration ends with

$$
\begin{aligned}
\cdots & \longrightarrow \pi_{2}\left(\Omega^{\Gamma}(X, x),(\gamma, g)\right) \longrightarrow \pi_{2}\left(\Lambda^{\Gamma} X,(\gamma, g)\right) \longrightarrow \pi_{2}(X, x) \longrightarrow \\
& \longrightarrow \pi_{1}\left(\Omega^{\Gamma}(X, x),(\gamma, g)\right) \longrightarrow \pi_{1}\left(\Lambda^{\Gamma} X,(\gamma, g)\right) \longrightarrow \pi_{1}(X, x) \longrightarrow \pi_{0} \longrightarrow \\
& \longrightarrow \pi_{0}\left(\Omega^{\Gamma}(X, x),(\gamma, g)\right) \longrightarrow \pi_{0}\left(\Lambda^{\Gamma} X,(\gamma, g)\right) \longrightarrow \pi_{0}(X, x)=\mathbf{1},
\end{aligned}
$$

where we have assumed that $X$ is path connected.

Since the topology on $\Gamma$ is discrete, we have $\pi_{0}\left(\Omega^{\Gamma}(X, x),(\gamma, g)\right)=$ $\pi_{1}^{\Gamma}(X, x)$, so that the last few terms of the sequence above become

$$
\begin{gathered}
\cdots \longrightarrow \pi_{1}\left(\Lambda^{\Gamma} X,(\gamma, g)\right) \longrightarrow \pi_{1}(X, x) \stackrel{\varepsilon}{\longrightarrow} \pi_{1}^{\Gamma}(X, x) \\
\longrightarrow \pi_{0}\left(\Lambda^{\Gamma} X,(\gamma, g)\right) \longrightarrow 1 .
\end{gathered}
$$

LEMMA 4.7. The map $\varepsilon$ is given by conjugation in $\pi_{1}^{\Gamma}(X, x)$ by elements of $\pi_{1}(X, x)$ :

$$
\varepsilon(\eta)=(\eta, e)^{-1}(\gamma, g)(\eta, e)=(\bar{\eta} * \gamma *(g \eta), g),
$$

where $\bar{\eta}$ is the reverse path of $\eta$.

Proof. The map $\varepsilon: \pi_{1}(X, x) \rightarrow \pi_{0}\left(\Omega^{\Gamma}(X, x),(\gamma, g)\right)=\pi_{1}^{\Gamma}(X, x)$ is the effect of lifting a loop $\eta \in \Omega(X, x)$ in the fibration (4.8). Let $t_{0} \in[0,1]$, and put $y=\eta\left(t_{0}\right)$. The $g$-loop $\bar{\eta}_{y} * \gamma *\left(g \cdot \eta_{y}\right)$, where $\eta_{y}(t)=\eta\left(t_{0} t\right)$ for $t \in[0,1]$ (see figure below), provides a deformation of $\gamma$, continuous in $t_{0}$, with base point $y$. Letting $t_{0}$ increase until $t_{0}=1$ gives the required expression.

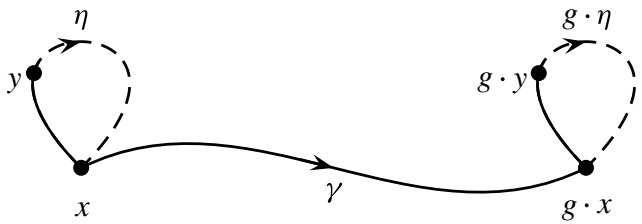

It follows from the exact sequence (4.10) that two $g$-loops $\gamma, \delta \in \Omega^{g}(X, x)$ are in the same connected component of $\Lambda^{g} X$ if and only if there is a loop $\eta \in$ 
$\Omega(X, x)$ and a homotopy

$$
\delta \sim \bar{\eta} * \gamma *(g \eta)
$$

If we assume that $X$ is aspherical, which will be true of the applications to choreographies, then we can easily deduce more (a space is aspherical if its universal cover is contractible; these are also known as Eilenberg-MacLane $K(\pi, 1)$ spaces). In this case, $\pi_{k}(X, x)=1$ for $k \geq 2$, and it follows that $\pi_{k}\left(\Omega^{\Gamma}(X, x)\right)=\mathbf{1}$ for $k \geq 1$, and the long exact sequence above then implies that $\pi_{k}\left(\Lambda^{\Gamma} X\right)=\mathbf{1}$ for $k \geq 2$. In particular, the '...' at the start of (4.10) can be replaced by 1 , and so $\pi_{1}\left(\Lambda^{\Gamma} X,(\gamma, g)\right)$ is isomorphic to the kernel of $\varepsilon$.

The preceding discussion can be summarized in the following statement, which is a restatement in terms of the equivariant fundamental group of two theorems in [26]. The theorem will be applied via Proposition 4.2 to spaces of symmetric loops.

THEOREM 4.8. Suppose that $X$ is a manifold with an action of a group $\Gamma$. For each $g \in \Gamma$, the relative loop space $\Lambda^{g} X$ enjoys the following properties.

(1) The connected components of $\Lambda^{g} X$ are in one-to-one correspondence with the set of orbits of the action of $\pi_{1}(X, x)$ on $\beta^{-1}(g) \subset \pi_{1}^{\Gamma}(X, x)$ by conjugation.

(2) If in addition $X$ is aspherical, then the connected component of $\Lambda^{g} X$ containing $\gamma$ is aspherical with fundamental group isomorphic to the 'partial centralizer'

$$
Z_{\pi_{1}^{\Gamma(X, x)}}((\gamma, g)) \cap \pi_{1}(X, x),
$$

where $Z_{\pi_{1}^{\Gamma}(X, x)}((\gamma, g))$ is the centralizer of the element $(\gamma, g)$ in $\pi_{1}^{\Gamma}(X, x)$.

Proof. (1) The exact sequence (4.10) together with Lemma 4.7 shows that two $g$-loops $\gamma, \delta \in \Omega^{g}(X, x)$ belong to the same connected component of $\Lambda^{g} X$ if and only if there exists $\eta \in \pi_{1}(X, x)$ such that

$$
(\delta, g)=(\eta, e)^{-1}(\gamma, g)(\eta, e) .
$$

That is, if and only if $(\gamma, g)$ and $(\delta, g)$ lie in the same orbit of the action of $\pi_{1}(X, x)$ on the coset $\beta^{-1}(g) \subset \pi_{1}^{\Gamma}(X, x)$. Different connected components therefore correspond to different orbits of this action.

(2) Since $X$ is aspherical, we have that $\pi_{k}\left(\Omega^{\Gamma}(X, x),(g, \gamma)\right)=0$ for $k \geq 1$, so the long exact sequence (4.9) shows that $\pi_{k}\left(\Lambda^{\Gamma} X,(\gamma, g)\right)=0$ for $k \geq 2$, and $\pi_{1}\left(\Lambda^{\Gamma} X,(\gamma, g)\right)$ is isomorphic to $\operatorname{ker} \varepsilon$. By Lemma 4.7, this kernel is the required 'partial centralizer'.

Before turning to the case in hand of $n$-body choreographies, we illustrate the theorem with two examples. 
EXAMPLE 4.9 (Taken from [26]). Consider $\Gamma=\mathbb{Z}_{2}=\left\langle\kappa \mid \kappa^{2}=e\right\rangle$ acting on $X=\mathbb{T}$ by $\kappa \theta=-\theta$. Then, using the fixed point $\theta=0$ as base point, one sees that $\pi_{1}^{\Gamma}(\mathbb{T}, 0)=\mathbb{Z} \rtimes \mathbb{Z}_{2}$, with $(a, \kappa)(b, \kappa)=(a-b, e)$. Thus $(a, e)(b, \kappa)(a, e)^{-1}=$ $(b+2 a, \kappa)$. It follows that, with $\tau(\kappa)=1 / 2$, we have $\operatorname{Fix}\left(\Gamma_{\tau}, \Lambda X\right) \simeq \Lambda^{\kappa} X$, and this has two connected components, determined by the parity of $b \in \mathbb{Z}$ in $(b, \kappa)$; both components are contractible.

EXAMPLE 4.10. For a more interesting example, let $X$ be the cylinder $\mathbb{T} \times \mathbb{R}$ with a single puncture at $(1 / 2,0)$. The fundamental group is the free group on two generators $F_{2}$. Choosing $x=(0,0)$ as a base point, the generators are the two loops $a$ and $b$, one above the puncture and one below, both chosen to go round the circle $\mathbb{T}$ once in the positive direction. Let $\Gamma=\mathbb{Z}_{2}$ act by reflection in the equator, so $\kappa \cdot(\theta, r)=(\theta,-r)$. Since $x$ is fixed by this action, we have that $\pi_{1}^{\Gamma}(X, x) \simeq F_{2} \rtimes \mathbb{Z}_{2}$, with $\mathbb{Z}_{2}$ acting on $F_{2}$ by $\kappa a=b$ and $\kappa b=a$. If we let $\tau: \Gamma \rightarrow \mathrm{S}^{1}$ be the only nontrivial homomorphism (so $\tau(\kappa)=(1 / 2)$ ), we have that

$$
\operatorname{Fix}\left(\Gamma_{\tau}, \Lambda X\right) \simeq \Lambda^{\kappa} X,
$$

and the latter space has connected components in one-to-one correspondence with the orbits of the action of $F_{2}$ on $\beta^{-1}(\kappa)<\pi_{1}^{\Gamma}(X, x)$. This action is

$$
(w, e)(z, \kappa)\left(w^{-1}, e\right)=\left(w z \bar{w}^{-1}, \kappa\right),
$$

where $w$ is any element of $F_{2}$, so any word in $a, b$, and $\bar{w}$ is the same word but with $a$ replaced everywhere by $b$ and vice versa. Thus two $\kappa$-loops $z, z^{\prime}$ based at $x$ are in the same connected component of $\operatorname{Fix}\left(\Gamma_{\tau}, \Lambda X\right)$ if and only if there is a $w \in F_{2}$ such that $z^{\prime}=w z \bar{w}^{-1}$. For example, all $\kappa$-loops of the form $w \bar{w}^{-1}$ are in the same connected component as the trivial loop at $x$. Moreover, that connected component is contractible by Theorem 4.8(2), since it is aspherical with fundamental group $\left\{w \in F_{2} \mid w \bar{w}^{-1}=e\right\}$, which is the trivial group. On the other hand, the connected component containing the $\kappa$-loop $a$ is not contractible, as $a b(a)(\overline{a b})^{-1}=a b(a) a^{-1} b^{-1}=a$, so all powers of $a b$ lie in the partial centralizer $Z_{F_{2} \rtimes Z_{2}}((a, \kappa)) \cap F_{2}$.

REMARK 4.11. The theorem above was written in terms of 'twisted conjugacy' in [26] (also known as Reidemeister conjugacy). Let $\pi$ be a group, and let $\phi$ be an endomorphism of $\pi$. Two elements $\gamma, \gamma^{\prime} \in \pi$ are said to be $\phi$-twisted conjugate if there is a $\delta \in \pi$ such that $\gamma^{\prime}=\delta \gamma \phi\left(\delta^{-1}\right)$. In the first example above $\phi(a)=-a$ (additively), and in the second $\phi(w)=\bar{w}$.

The description in [26] is related to the present approach as follows. For $g \in \Gamma$, let $\omega=\omega_{g}$ be a fixed path from $x$ to $g \cdot x$. Then we can identify the coset $\beta^{-1}(g) \subset \pi_{1}^{\Gamma}(X, x)$ with $\pi_{1}(X, x)$ by making $\zeta \in \pi_{1}(X, x)$ correspond to $(\zeta, e)\left(\omega_{g}, g\right)=\left(\zeta * \omega_{g}, g\right)$ in $\beta^{-1}(g)$. The expression for $\varepsilon$ in (4.11) above using 
this identification is, for $\eta \in \pi_{1}(X, x)$,

$$
\varepsilon(\eta)=\bar{\eta} *\left(\zeta * \omega_{g}\right) *(g \eta) * \overline{\omega_{g}} .
$$

Thus $\varepsilon(\eta)=\eta^{-1} \zeta \phi(\eta)$, which is precisely Reidemeister conjugation of $\zeta$, where $\phi$ is the automorphism

$$
\phi(\eta)=\omega_{g} *(g \eta) * \overline{\omega_{g}} .
$$

Further details can be found in [37, Section 2.5].

\section{Choreographies and braids}

We now apply the results of the previous section to the case in hand of $n$ distinct points in the plane, and in particular to the choreographic loops. We are interested in the action of finite subgroups $G$ of $\Gamma=\mathrm{O}(2) \times S_{n}$ on $X^{(n)}$, and we use some of the properties above to find the equivariant fundamental group $\pi_{1}^{G}\left(X^{(n)}, x\right)$. (We ignore the temporal part $\mathrm{S}^{1}$ here, as its action on $X^{(n)}$ is trivial.)

5.1. Configuration space and braids. First, we recall some facts about braid groups, and introduce some notation. Two useful references are the books by Kessel and Turaev [24] and Farb and Margalit [11]. It was first observed by Fox and Neuwirth [14] that the fundamental group of the space $X^{(n)}$ is (isomorphic to) the pure braid group $P_{n}$, and it was moreover proved by Fadell and Neuwirth [10] that $X^{(n)}$ is aspherical. Recall that the braid groups sit in a short exact sequence

$$
\mathbf{1} \rightarrow P_{n} \longrightarrow B_{n} \stackrel{\pi}{\longrightarrow} S_{n} \rightarrow \mathbf{1},
$$

where $B_{n}$ is the full braid group on $n$ strings, and $S_{n}$ the permutation group. Furthermore, $S_{n}$ acts freely on $X^{(n)}$, and the fundamental group of the quotient space $X^{(n)} / S_{n}$ is the full braid group $B_{n}$ (as observed in [14]). It follows that the equivariant fundamental group $\pi_{1}^{S_{n}}\left(X^{(n)}, x\right)$ is isomorphic to the braid group $B_{n}$, and that (4.3) becomes (5.1) (that is, $\beta$ becomes $\pi$ ). Here, we are taking the usual base point considered for braid groups, namely $x$ is any point in $X^{(n)}$ where the $z_{j}$ are placed in sequence along the real line.

Let us denote the generators of the braid group $B_{n}$ by $\mathfrak{b}_{1}, \mathfrak{b}_{2}, \ldots, \mathfrak{b}_{n-1}$, where $\mathfrak{b}_{i}$ represents the crossing of string $i+1$ over (in front of) string $i$ (these are usually denoted $\sigma_{1}, \ldots, \sigma_{n-1}$, but this would conflict with our use of $\sigma_{i}$ as permutations). In terms of the motion of points in the plane, $\mathfrak{b}_{i}$ represents the clockwise interchange of points $i$ and $i+1$. The centre of both groups $B_{n}$ and $P_{n}$ is infinite cyclic, generated by the full twist $\Delta^{2}$, where

$$
\Delta=\left(\mathfrak{b}_{1} \mathfrak{b}_{2} \ldots \mathfrak{b}_{n-1}\right)\left(\mathfrak{b}_{1} \mathfrak{b}_{2} \ldots \mathfrak{b}_{n-2}\right) \ldots\left(\mathfrak{b}_{1}\right)
$$

The full twist represents a clockwise rotation through $2 \pi$ of the whole braid. 


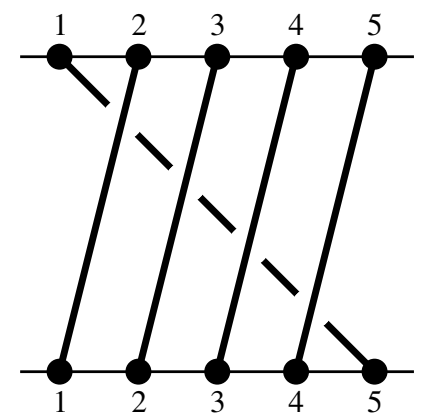

Figure 9. The braid $\delta$ for $n=5$. We have $\pi(\delta)=\left(\begin{array}{lllll}1 & 2 & 3 & 4 & 5\end{array}\right)=\sigma_{1}$.

Denote by $\delta$ the element $\delta=\mathfrak{b}_{1} \mathfrak{b}_{2} \ldots \mathfrak{b}_{n-1}$ (see Figure 9). In the exact sequence (5.1), we have $\pi(\delta)=\sigma_{1}=(12 \ldots n)$. It follows that $\delta^{n} \in P_{n}$, and in fact $\delta^{n}=\Delta^{2}$ (as is not hard to see geometrically).

REMARK 5.1. Some care should taken to express correctly the permutation associated to a given braid, since we are using the usual left action of the permutation group. Now, as homotopy classes of curves in $X^{(n)}$, the product $\mathfrak{b} \mathfrak{b}^{\prime}$ is the class $\left[\mathfrak{b} *\left(\sigma \cdot \mathfrak{b}^{\prime}\right)\right]$, where $\sigma=\pi(\mathfrak{b})$. It follows that $\pi\left(\mathfrak{b} \mathfrak{b}^{\prime}\right)=\pi(\mathfrak{b}) \circ \pi\left(\mathfrak{b}^{\prime}\right)$ (so first applying $\pi\left(\mathfrak{b}^{\prime}\right)$ on $X^{(n)}$ and then $\pi(\mathfrak{b})$ - this is consistent with the usual relation between free group actions and fundamental groups, as described for example in Spanier [36, Ch. 2]). In particular, the permutation associated to the

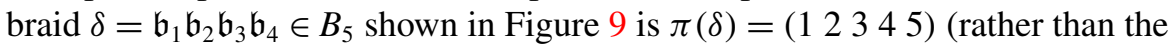
usual ( $\left(\begin{array}{lllll}5 & 4 & 3 & 2 & 1\end{array}\right)$ ).

There is a homomorphism $\chi: B_{n} \rightarrow \mathbb{Z}$, generated by $\chi\left(\mathfrak{b}_{i}\right)=1$ (for all $i$ ), and called the exponent sum of the braid; it measures the algebraic number of crossings in the braid. For example, $\chi(\delta)=n-1$, while $\chi(\Delta)=\frac{1}{2} n(n-1)$. The important property in the present context is that $\chi$ is invariant under conjugation, as is easy to see. (More abstractly, the first homology group of $B_{n}$ is $\mathbb{Z}$, and $\chi$ is the natural projection $B_{n} \rightarrow H_{1}\left(B_{n}\right) \simeq B_{n} /\left[B_{n}, B_{n}\right]$.)

We define two subgroups of the braid group which will be useful when we consider choreographies with nontrivial core below.

DEFinitions 5.2. First, we let $B_{n, 1}$ be the group

$$
B_{n, 1}=\left\{\mathfrak{b} \in B_{n+1} \mid \pi(\mathfrak{b})(0)=0\right\},
$$

where the $n+1$ strings are labelled $0,1, \ldots, n$. Its generators are described in the review by Vershinin [40] (where $B_{n, 1}$ is denoted $B r_{1, n+1}$ ). 
Second, we define a subgroup $P_{n, 1(c)}$ of $P_{n+1}$ as follows (again label the strings $0, \ldots, n)$. For each pure braid $p \in P_{n+1}$ there are integers $w_{i j}(p)$ given by the number of times string $i$ winds around string $j$. Then put

$$
P_{n, 1(c)}=\left\{p \in P_{n+1} \mid w_{j 0}(p) \equiv 0(\bmod c), \forall j=1 \ldots, n\right\} .
$$

That is, the number of times each string winds around the 0 -string is an integer multiple of $c$. (This also has a homological interpretation, as the winding numbers of a loop are the components of the image of the element of $P_{n}$ in its first homology group: $H_{1}\left(P_{n}\right) \simeq \mathbb{Z}^{n(n-1) / 2}$.)

Recall that $\sigma_{1} \in S_{n}$ is the permutation $\sigma_{1}(j)=j+1(\bmod n)$.

Proposition 5.3. Suppose that $c$ divides $n$, with $1<c<n$, and that $\ell$ is coprime to $c$. Let $K$ be the subgroup of $\Gamma$ generated by $\left(R_{2 \pi \ell / c}, \sigma_{1}^{\widehat{n}}\right)$, where $\widehat{n}=n / c$, so $K$ is cyclic of order $c$. The space $Y=\operatorname{Fix}\left(K, X^{(n)}\right)$ is connected and aspherical, with fundamental group $\pi_{1}(Y, y) \simeq P_{\widehat{n}, 1(c)}<P_{\widehat{n}+1}$.

Proof. We saw in Section 3.2 that $Y$ is a cover of $X_{*}^{(\widehat{n})}$, of degree $c^{\widehat{n}}$. The homotopy type of $X_{*}^{(\widehat{n})}$ is found from the fibration $X^{(\widehat{n}+1)} \rightarrow \mathbb{C}$ given by

$$
\left(z_{0}, z_{1}, \ldots, z_{\widehat{n}}\right) \longmapsto z_{0} .
$$

The fibre over 0 is $X_{*}^{(\hat{n})}$, and, since the base is contractible, the total space and the fibre are homotopic. That is, $X_{*}^{(\widehat{n})} \sim X^{(\widehat{n}+1)}$, and the latter is aspherical with fundamental group $P_{\widehat{n}+1}$, as already pointed out. Now consider the covering $\Psi: Y \rightarrow X_{*}^{(\widehat{n})}$ of degree $c^{\widehat{n}}$ given in (3.3), which is in fact the quotient map for the action of $\mathbb{Z}_{c}^{\widehat{n}}$ acting by multiplication by $c$ th roots of unity:

$$
\left(\omega_{1}, \ldots, \omega_{\hat{n}}\right) \cdot\left(z_{1}, \ldots, z_{n}\right)=\left(\omega_{1} z_{1}, \ldots, \omega_{\hat{\jmath}} z_{j}, \ldots, \omega_{\hat{n}} z_{n}\right) \quad\left(\omega_{j} \in \mathbb{Z}_{c}\right),
$$

where $\widehat{\jmath}=j(\bmod \widehat{n})$. This gives the short exact sequence,

$$
\mathbf{1} \rightarrow \pi_{1}(Y, x) \longrightarrow \pi_{1}\left(X_{*}^{(\widehat{n})}, \Psi(x)\right) \longrightarrow \mathbb{Z}_{c}^{\widehat{n}} \rightarrow \mathbf{1} .
$$

The projection to $\mathbb{Z}_{c}^{\widehat{n}}$ is the winding number modulo $c$ of each of the strings (particles) around the origin, so in order for a loop to be in $\pi_{1}(Y, x)$ these winding numbers must vanish, modulo $c$.

For the following theorem, we need to define an involution $\mathfrak{b} \mapsto \overline{\mathfrak{b}}$ on $B_{n}$ : given any braid $\mathfrak{b} \in B_{n}$, then $\overline{\mathfrak{b}}$ is the same braid but with every overcrossing changed into an undercrossing and vice versa. This is a homomorphism, and clearly this leaves the resulting permutation unaffected: $\pi(\overline{\mathfrak{b}})=\pi(\mathfrak{b})$. Algebraically, let $\mathfrak{b}$ be any word written in terms of the generators $\mathfrak{b}_{j}$; then $\overline{\mathfrak{b}}$ is the same word with each instance of a generator replaced by its inverse. It follows that the exponent sum satisfies $\chi(\overline{\mathfrak{b}})=-\chi(\mathfrak{b})$. For example, if $\mathfrak{b}=\mathfrak{b}_{i} \mathfrak{b}_{j}^{-2}$, then $\overline{\mathfrak{b}}=\mathfrak{b}_{i}^{-1} \mathfrak{b}_{j}^{2}$. One can show easily that $\bar{\Delta}=\Delta^{-1}$. 
Recall the notation $\times_{\alpha}$ and the curve $(-\theta)_{g \cdot x}$ defined in Example 4.4. Recall also that $R_{2 \pi \theta}$ is the rotation through $2 \pi \theta$ in the plane, and that $\kappa$ is the reflection in the $x$-axis (complex conjugation).

THEOREM 5.4. For $\Gamma=\mathrm{O}(2) \times S_{n}$ acting on $X^{(n)}$, with $x$ the usual base point for braids, there is an isomorphism

$$
\begin{aligned}
\pi_{1}^{\Gamma}\left(X^{(n)}, x\right) & \stackrel{\sim}{\longrightarrow}\left(B_{n} \times_{\alpha} \mathbb{R}\right) \rtimes \mathbb{Z}_{2} \\
(\gamma, g) & \longmapsto \begin{cases}\left(\left[\gamma *(-\theta)_{g \cdot x}, \theta\right], e\right) & \text { if } g=\left(R_{2 \pi \theta}, \sigma\right) \\
([\gamma, 0], \kappa) & \text { if } g=(\kappa, \sigma),\end{cases}
\end{aligned}
$$

where $\sigma \in S_{n}$. The image of the remaining elements, so those of the form $g=$ $\left(R_{2 \pi \theta} \kappa, \sigma\right)$, can be determined using the semidirect product structure coming from $\kappa[\mathfrak{b}, s]=[\overline{\mathfrak{b}},-s]$. The homomorphism $\alpha: \mathbb{Z} \rightarrow B_{n}$ is given by $\alpha(n)=\Delta^{2 n}$. In terms of this isomorphism, the projection $\beta: \pi_{1}^{\Gamma} \rightarrow \Gamma$ is given by

$$
\beta\left([\mathfrak{b}, \theta], \kappa^{r}\right)=\left(R_{2 \pi \theta} \kappa^{r}, \pi(\mathfrak{b})\right) \in \mathrm{O}(2) \times S_{n},
$$

where $\pi: B_{n} \rightarrow S_{n}$ is the usual projection.

Proof. We use Proposition 4.5 and Equation (4.4), with $N=S_{n} \triangleleft \mathrm{O}(2) \times S_{n}$. First, $S_{n}$ acts freely on $X^{(n)}$, so that

$$
\pi_{1}^{S_{n}}\left(X^{(n)}, x\right) \simeq \pi_{1}\left(X^{(n)} / S_{n}, \bar{x}\right) \simeq B_{n} .
$$

Now apply Proposition 4.5 to the $\mathrm{SO}(2)$-action to obtain (we identify $\mathrm{S}^{1} \stackrel{\sim}{\longrightarrow}$ $\mathrm{SO}(2)$ by $\left.\theta \mapsto R_{2 \pi \theta}\right)$

$$
\pi_{1}^{\mathrm{SO}(2) \times S_{n}}\left(X^{(n)}, x\right) \simeq \pi_{1}^{\mathrm{SO}(2)}\left(X^{(n)} / S_{n}, \hat{x}\right) \simeq \pi_{1}\left(X^{(n)} / S_{n}, \hat{x}\right) \times_{\alpha} \mathbb{R} .
$$

The second isomorphism, by Example 4.4, is $(\hat{\gamma}, \theta) \mapsto\left[\hat{\gamma} *(-\theta)_{\theta \cdot x}, \theta\right]$, where $\hat{\gamma}$ is a path in $X^{(n)} / S_{n}$ with phase $\theta$. Let $\gamma$ be the unique lift of the path $\hat{\gamma}$ to $X^{(n)}$ with base point $x$. Since $\hat{\gamma}(1)=\theta \cdot \hat{x}$, we have that $\gamma(1)=\sigma \cdot \theta \cdot x=\theta \cdot \sigma \cdot x$ for some $\sigma \in S_{n}$. That is, $\gamma(1)=g \cdot x$ for $g=\left(R_{2 \pi \theta}, \sigma\right) \in \mathrm{SO}(2) \times S_{n}$. Consequently, $\gamma *(-\theta)_{g \cdot x}$ is a path from $x$ to $\sigma \cdot x$, and so represents a braid, as required.

The final result then follows by using $\kappa \in \mathrm{O}(2)$-the reflection in the $x$-axis (real line) which fixes the base point and makes every overcrossing into an undercrossing and vice versa, so changing each $\mathfrak{b}_{j}$ into $\mathfrak{b}_{j}^{-1}$.

For the homomorphism $\beta$, the element $s \in \mathbb{R}$ corresponds to rotation through an angle of $2 \pi s$, and $\kappa$ corresponds to the reflection in the $x$-axis. The choice of semi-direct product structure then determines the first component of $\beta$. The second component $\pi(b)$ is determined by the projection associated to Equation (5.4) above. 
For future reference, if $p \in P_{n}$, which we consider as the element $(p, 0, e) \in$ $\left(B_{n} \times_{\alpha} \mathbb{R}\right) \rtimes \mathbb{Z}_{2}$, then conjugation by $p$ is given by

$$
p(\mathfrak{b}, \theta, e) p^{-1}=\left(p \mathfrak{b} p^{-1}, \theta, e\right) \quad \text { and } \quad p(\mathfrak{b}, \theta, \kappa) p^{-1}=\left(p \mathfrak{b} \bar{p}^{-1}, \theta, \kappa\right) .
$$

The first of these just involves the usual conjugacy in the braid group, while the second uses a twisted (Reidemeister) conjugacy: in the notation of Remark 4.11, it uses $\phi(p)=\bar{p}$.

EXAMPLE 5.5. The equivariant fundamental groups for the nonreversing symmetry groups without core are subgroups of the group $\pi_{1}^{\Gamma}\left(X^{(n)}, x\right)$ found in Theorem 5.4. For the cyclic group $\Sigma_{n}<S_{n}$ (the 'spatial component' of the choreography group $\mathfrak{C}_{n}$ ), one has

$$
\pi_{1}^{\Sigma_{n}}\left(X^{(n)}, x\right) \simeq C B_{n},
$$

a group we call the cyclic braid group, and which is the subgroup of $B_{n}$ generated by $P_{n}$ and $\delta$; it is of course the inverse image under $\pi: B_{n} \rightarrow S_{n}$ of the cyclic group $\Sigma_{n}$. The homomorphism $\beta: \pi_{1}^{\Sigma_{n}} \rightarrow \Sigma_{n}$ here is just the restriction of $\pi$ to this cyclic braid group.

Combining this example with the $\mathrm{SO}(2)$ action, we can find $\pi_{1}^{G}\left(X^{(n)}, x\right)$ for $G=C(n, k / \ell)$, using Example 4.4. Recall that, provided that $(n, k)=1, G$ is cyclic of order $n k$ and is generated by $g=\left(R_{2 \pi a \ell / k}, \sigma_{1}^{b}\right)$, where $a n-b k=1$.

Corollary 5.6. (1) Let $G=C(n, k / \ell)$, with $(n, k)=1$. There is an isomorphism

$$
\pi_{1}^{G}\left(X^{(n)}, x\right) \stackrel{\sim}{\longrightarrow} C B_{n} \times_{\alpha} \mathbb{Z}(1 / k),
$$

where $\mathbb{Z}(1 / k)$ is the subgroup of $\mathbb{R}$ consisting of integer multiples of $1 / k$. Given $(\gamma, g) \in \pi_{1}^{G}(X, x)$ for $g=\left(R_{2 \pi a \ell / k}, \sigma_{1}^{b}\right)$, the corresponding element of the right-hand side is

$$
\left[\gamma *(-\theta)_{g \cdot x}, a \ell / k\right],
$$

for $\theta=a \ell / k \in \mathbb{Z}(1 / k)$ (the path $\theta_{p}$ is defined in Example 4.4). Under this isomorphism, the projection $\beta: \pi_{1}^{G} \rightarrow G<\mathrm{SO}(2) \times \Sigma_{n}$ is given by

$$
\beta([\mathfrak{b}, \theta])=\left(R_{2 \pi \theta}, \pi(\mathfrak{b})\right) .
$$

(2) Let $G=C^{\prime}(n, 2)$, which is cyclic of order $2 n$, and its projection to $\Gamma$ is generated by $\left(\kappa, \sigma_{2}\right) \in \Gamma$. There is an isomorphism

$$
\pi_{1}^{G}\left(X^{(n)}, x\right) \stackrel{\sim}{\longrightarrow} C B_{n} \rtimes \mathbb{Z}_{2},
$$

where the semidirect product comes from $\kappa \cdot \mathfrak{b}=\overline{\mathfrak{b}}$. Any relative loop $\gamma$ with $g=\left(\kappa, \sigma_{2}\right)$ maps under the isomorphism to $(\gamma, \kappa)$, and $\beta\left(\gamma, \kappa^{r}\right)=\left(\kappa^{r}, \pi(\gamma)\right)$. 


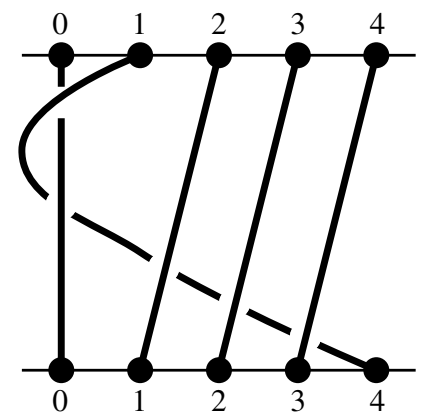

Figure 10. The braid $\widehat{\delta} \in B_{\widehat{n}, 1}$ for $G=C(n, k / \ell)$ with $(n, k)=c>1$, shown here for $\widehat{n}=4$; note that $\pi(\widehat{\delta})=\left(\begin{array}{llll}1 & 2 & 3 & 4\end{array}\right)=\widehat{\sigma}_{1}$. See Remark 5.1

Proof. Since the groups are subgroups of $\Gamma$, these statements are all immediate consequences of the more general Theorem 5.4.

5.2. Choreographies. We now return to the case of choreographies, where the equivariant loops are those arising from the classification of Section 2. Since we are looking at nonreversing symmetry groups in this section, there are two cases: $C(n, k / \ell)$ and, if $n$ is odd, $C^{\prime}(n, 2)$, defined in Section 2.3. (We consider the time-reversing symmetries in a separate paper.)

Recall that for $C(n, k / \ell)$ we denote the core by $K$ : it is the pointwise isotropy of a loop with this symmetry, and is the cyclic group of order $c=(n, k)$ generated by $\left(R_{2 \pi \ell / c}, \sigma_{1}^{\widehat{n}}\right) \in \Gamma$. If $c=1$, or if $G=C^{\prime}(n, 2)$, then $K$ is trivial.

Combining Propositions 4.2, 5.3, and Theorem 4.8, we deduce the main result of this section. For the part with nontrivial core, it will be useful to use the subgroup $B_{\widehat{n}, 1}$, defined in Equation (5.2). Note that the projection $\pi: B_{\widehat{n}+1} \rightarrow$ $S_{\widehat{n}+1}$ restricts to $B_{\widehat{n}, 1} \rightarrow S_{\widehat{n}}$. Define the element $\widehat{\delta}=\mathfrak{b}_{0}^{2} \mathfrak{b}_{1} \mathfrak{b}_{2} \ldots \mathfrak{b}_{\widehat{n}-1} \in B_{\widehat{n}, 1}$ (see Figure 10), and note that $\pi(\widehat{\delta})=\widehat{\sigma}_{1}=(12 \ldots \widehat{n}) \in S_{\widehat{n}+1}$. In fact, though we do not use it, it is well known that $(\widehat{\delta})^{\widehat{n}-1}=\Delta^{2}$.

THEOREM 5.7. Let $G$ be any of the symmetry groups $C(n, k / \ell)$ or $C^{\prime}(n, 2)$.

(1) The set of connected components of the space $\operatorname{Fix}\left(G, \Lambda X^{(n)}\right)$ is in one-to-one correspondence with the following sets.

- For $G=C(n, k / \ell)$ with $(n, k)=1$, the set of $P_{n}$-conjugacy classes in the coset $\delta^{b} P_{n} \subset C B_{n}$, where $b k \equiv-1(\bmod n)$.

- For $G=C(n, k / \ell)$ with $(n, k)=c>1$, the set of $P_{\widehat{n}, 1(c)}$-conjugacy classes in the coset $\widehat{\delta}^{b} P_{\widehat{n}, 1(c)} \subset B_{\widehat{n}, 1}$, where $\widehat{\delta} \in B_{\widehat{n}, 1}$ is defined above, and $b k \equiv$ $-c(\bmod n)$. 
- For $G=C^{\prime}(2, n)$, the set of orbits of the twisted conjugacy action of $P_{n}$ on the coset $\delta^{\prime} P_{n}$, where $\delta^{\prime}=\delta^{(n+1) / 2}$, and the twisted conjugacy action is $p \cdot \mathfrak{b}=p \mathfrak{b} \bar{p}^{-1}$, for $p \in P_{n}$, and the definition of $\bar{p}$ precedes Theorem 5.4.

(2) The connected component containing $u \in \operatorname{Fix}(G, \Lambda X)$ is aspherical with fundamental group isomorphic to the group $\Pi_{u}$ defined as follows.

- For $G=C(n, k / \ell)$ with $(n, k)=1$, then

$$
\Pi_{u}=\left\{p \in P_{n} \mid p \mathfrak{b}=\mathfrak{b} p\right\},
$$

where $\mathfrak{b} \in \delta^{b} P_{n}<B_{n}$ is the braid corresponding to the path $t \mapsto u(t / n k)$ for $t \in[0,1]$ and $b k \equiv-1(\bmod n)$.

- For $G=C(n, k / \ell)$ with $(n, k)=c>1$ it is the analogue, with $X^{(n)}$ replaced by $Y$, and so $p \in P_{\widehat{n}, 1(c)}$ in place of $P_{n}$,

$$
\Pi_{u}=\left\{p \in P_{\widehat{n}, 1(c)} \mid p \mathfrak{b}=\mathfrak{b} p\right\},
$$

where $\mathfrak{b} \in \widehat{\delta}^{b} P_{\widehat{n}, 1(c)}<B_{\widehat{n}, 1}$ is the braid corresponding to the path $t \mapsto u(c t / n k)$ for $t \in[0,1]$ and $b k \equiv-c(\bmod n)$.

- $\operatorname{For} C^{\prime}(n, 2)$, it is

$$
\Pi_{u}=\left\{p \in P_{n} \mid p \mathfrak{b}=\mathfrak{b} \bar{p}\right\},
$$

where $\mathfrak{b} \in B_{n}$ is the braid corresponding to the path $t \mapsto u(t / 2 n)$ for $t \in[0,1]$.

For example, for choreographies with symmetry $\mathfrak{C}=C(n, 1)$, we have that $k=1$, so $b=-1$. The relevant coset is therefore $\delta^{-1} P_{n}$.

REMARKS 5.8. (a) It is easy to see that each of the cosets occurring in part (1) contains infinitely many conjugacy classes, since multiplication by powers of $\Delta^{2}$ preserves the coset, but changes the conjugacy class, as is readily seen by considering the exponent sum (which is invariant under conjugacy).

(b) Note that in the theorem the $a, b$ are not unique. However, different choices of $b$ differ by multiples of $n$, and $\delta^{b+r n}=\delta^{b} \Delta^{2 r}$. So first the cosets $\delta^{b} P_{n}$ and $\delta^{b+r n} P_{n}$ coincide, and second, while the conjugacy classes are not the same, they are in (natural) one-to-one correspondence, because $\Delta^{2}$ is central.

(c) Finding centralizers of braid elements is an interesting problem in braid theory; see for example [19]. However, the known results do not address the twisted conjugacy problem relevant to $C^{\prime}(n, 2)$, although some general results do exist [18].

Proof. By Proposition 4.2, we know that $\operatorname{Fix}\left(G, \Lambda X^{(n)}\right)$ is homeomorphic to $\Lambda^{g} X^{(n)}$ if the core is trivial, and to $\Lambda^{g} Y=\Lambda^{g} Y_{K}$ if the core $K$ is nontrivial, for some suitable $g \in \Gamma$ discussed below. In each case, given a loop $u \in \operatorname{Fix}\left(G, X^{(n)}\right)$, 
one defines a suitable $g$-loop $\gamma$ according to Proposition 4.2. The set of connected components is then given in part (1) of Theorem 4.8, while the fundamental group of each component is given in part (2) of the same theorem. We proceed by treating each class of symmetry group in turn: first the two cases with trivial core, and then the remaining groups with nontrivial core, which requires a more involved argument.

First, suppose that $G=C(n, k / \ell)$, with $(n, k)=1$. Here, $G$ is cyclic of order $n k$, and its projection to $\Gamma$ is generated by $g=\left(R_{2 \pi а \ell / k}, \sigma_{1}^{b}\right) \in \Gamma$, with $a n-b k=1$. Now $\pi_{1}^{G}$ is given in Corollary 5.6, and $(\gamma, g)$ represents an element $[\mathfrak{b}, r / k] \in C B_{n} \times_{\alpha} \mathbb{Z}(1 / k) \simeq \pi_{1}^{G}$ for some $\mathfrak{b} \in B_{n}$ and $r \in \mathbb{Z}$. Then $\beta([\mathfrak{b}, r / k])=$ $\left(R_{2 \pi r \ell / k}, \pi(\mathfrak{b})\right) \in \mathrm{SO}(2) \times S_{n}$, from which $r=a$ and $\pi(\mathfrak{b})=\sigma_{1}^{b}$, and consequently $\mathfrak{b}$ belongs to the coset $\delta^{b} P_{n}$. The final statement in this case then follows from the expression for conjugation given in (5.6).

Next, suppose that $u$ has symmetry $G=C^{\prime}(n, 2)$, which is the cyclic group of order $2 n$ whose projection to $\Gamma$ is generated by $g^{\prime}=\left(\kappa, \sigma_{2}\right)$ (recall that $n$ is odd, so $\sigma_{2}=\sigma_{1}^{h}$ with $\left.h=(n+1) / 2\right)$. Then $\beta^{-1}\left(g^{\prime}\right) \subset \pi_{1}^{\Gamma}\left(X^{(n)}, x\right)$ is the coset $\left(\delta^{h} P_{n}, 0, \kappa\right)<\pi_{1}^{\Gamma}\left(X^{(n)}, x\right)$ (see Theorem 5.4 for notation). It then follows from the second part of (5.6) that the conjugation by $p \in P_{n}$ is given by $\mathfrak{b} \mapsto p \mathfrak{b} \bar{p}^{-1}$.

Finally, suppose that $G$ has nontrivial core, that is $G=C(n, k / \ell)$ with $(n, k)=$ $c>1$. Then, by Proposition 4.2, we have that $\operatorname{Fix}\left(G, \Lambda X^{(n)}\right) \simeq \Lambda^{g} Y$ for $g=$ $\left(R_{2 \pi a \ell / k}, \sigma_{1}^{b}\right)$, with $a n-b k=c$; see (2.4). By Proposition 5.3, the fundamental group of $Y=\operatorname{Fix}\left(K, X^{(n)}\right)$ is $P_{\widehat{n}, 1(c)}$, so that the connected components of $\Lambda^{g} Y$ are in one-to-one correspondence with the $P_{\widehat{n}, 1(c)}$-conjugacy classes in the coset $\beta^{-1}(g) \subset \pi_{1}^{N / K}(Y, x)$, where $N$ is any group containing $K$ and $g$ and acting on $Y$. It remains to identify a suitable group $N$ so that the commutation is as given in the theorem.

We take the group $N$ as follows. The core $K$ is generated by $\left(R_{-2 \pi \ell(c)}, \sigma_{1}^{\widehat{n}}\right)$, and $\sigma_{1}^{\widehat{n}}$ is a product of $\widehat{n}$ disjoint cycles of length $c$. For $j=1, \ldots, \widehat{n}$, let $\pi_{j} \in S_{n}$ be the cycle of length $c$,

$$
\pi_{j}=(j(\widehat{n}+j)(2 \widehat{n}+j) \cdots((c-1) \widehat{n}+j)) ;
$$

that is, $\pi_{j}(j+r \widehat{n})=j+(r+1) \widehat{n}(\bmod n)$, for $r=0 \ldots c-1$. Then, as a product of disjoint cycles, $\sigma_{1}^{\widehat{n}}=\pi_{1} \pi_{2} \ldots \pi_{\widehat{n}}$. Let $N<N_{\Gamma}(K)$ be

$$
N=\mathrm{SO}(2) \times\left(\mathbb{Z}_{c}^{\widehat{n}} \rtimes S_{\widehat{n}}\right) .
$$

The action on $Y$ is by $\mathrm{SO}(2)$ acting on the plane as usual, the $j$ th component of $\mathbb{Z}_{c}^{\widehat{n}}$ acting by powers of the cycle $\pi_{j}$ (equivalently, on $Y$ by multiplication by $c$ th roots of unity as in (5.3)), and $S_{\widehat{n}}$ acting by permuting the $\widehat{n}$ disjoint cycles in $\sigma_{1}^{\widehat{n}}$. (The permutation part of $N$ is the wreath product $\mathbb{Z}_{c}>S_{\widehat{n}}$.) Clearly $K<N$, and there is therefore an action of $N / K$ on $Y$. Moreover, the subgroup $\mathbb{Z}_{c}^{\widehat{n}}$ acts freely, and the quotient $Y / \mathbb{Z}_{c}^{\widehat{n}}$ can be identified with $X_{*}^{(\widehat{n})}$ via the map $\Psi$ defined in (3.3). 


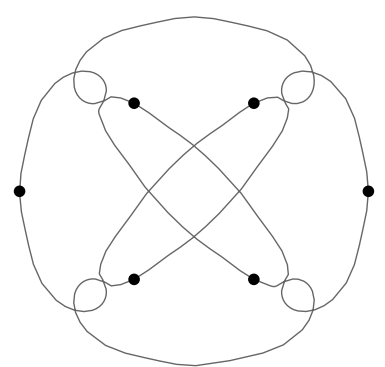

(a) $D(6,4)$

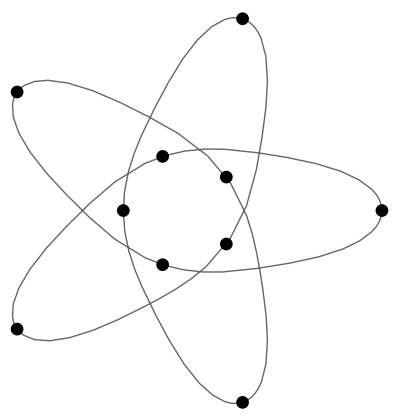

(b) $D(10,5 / 2)$

Figure 11. Two examples illustrating different connected components of each symmetry type compared with those in Figure 5; see also Remark 1.6. Many examples of five-body choreographies with a single time-reversing reflectional symmetry-so type $D(5,1)$-in different connected components have been found (numerically) by Simó [35, Figure 3].

Let $\widehat{\Gamma}=\mathrm{SO}(2) \times S_{\widehat{n}}$ act in the usual way on $X_{*}^{(\widehat{n})}$, and let $\psi: N / K \rightarrow \widehat{\Gamma}$ be the surjective homomorphism $\psi(R, \sigma)=\left(R^{c}, \widehat{\sigma}\right)$, where $\widehat{\sigma}(j)=\sigma(J)(\bmod \widehat{n})$. The kernel of $\psi$ is precisely $\mathbb{Z}_{c}^{\widehat{n}}$, and the map $\Psi$ is then equivariant with respect to $\psi$, meaning that $\Psi(g \cdot x)=\psi(g) \cdot \Psi(x)$.

Since the $\mathbb{Z}_{c}^{\widehat{n}}$-action is free, by Proposition 4.5 we have an isomorphism

$$
\pi_{1}^{N / K}(Y, x) \simeq \pi_{1}^{\widehat{\Gamma}}\left(X_{*}^{(\widehat{n})}, x\right)=B_{\widehat{n}, 1} \times_{\alpha} \mathbb{R},
$$

where $\alpha(1)=\widehat{\Delta}^{2}$, where $\widehat{\Delta}^{2}$ is the full twist in $P_{\widehat{n}+1} \subset B_{\widehat{n}, 1}$ (the proof of the final equality in (5.10) is the same as that for Theorem 5.4, bearing in mind that only $S_{\widehat{n}}$ acts and not $S_{\widehat{n}+1}$, and we are not including the reflection $\left.\kappa\right)$. Now, with $\widehat{\delta} \in B_{\widehat{n}, 1}$ defined just before the statement of the theorem, we have that $\pi(\widehat{\delta})=\widehat{\sigma}_{1}$, so (similar to the case above)

$$
\beta^{-1}\left(R_{2 \pi a \ell / k}, \sigma_{1}^{b}\right)=\widehat{\delta}^{b} P_{\widehat{n}, 1(c)},
$$

as required, where $\beta: \pi_{1}^{N / K}(Y, x) \rightarrow N / K$.

The fundamental group of the component containing $u$ is then deduced in the same way as before.

Choreographies with the same symmetry but in different connected components can often be distinguished by their sequence of winding numbers: since the particles move without collision, in the full periodic orbit each pair of particles winds around each other some integer number of times. For a given choreography, the winding number for particles $i$ and $j$ depends only on the difference $|i-j|$, because of the choreography symmetry. For the two 
choreographies depicted in Figure 11 the sequence of winding numbers is $(-3,-3,1)$ and $(-3,-3,2,-3,-3)$, respectively, while for the choreographies of Figure 5 they are $(1,-3,1)$ and $(-3,2,2,2,-3)$, respectively. The two with symmetry $D(6,4)$ have different winding numbers, and so are not homotopic; similarly for the two $D(10,5 / 2)$-choreographies. Here, the notation is that the $k$ th term of the sequence is the winding number of particle $j$ around particle $j+k$. It is not hard to show that, for choreographies of type $C(n, k, \ell)$, the winding numbers are all equivalent to $\ell(\bmod k)$.

EXAMPLE 5.9. Consider the circular choreography $u$, with speed $\ell=1$ and $n$ particles (Example 1.3). If $z_{0}=\left(\omega, \omega^{2}, \ldots, \omega^{n-1}, 1\right) \in X^{(n)}$, where $\omega=e^{2 \pi i / n}$, then $u(t)=e^{2 \pi i t} z_{0}$. For this example, it is convenient to use $z_{0}$ as the base point. The generators of the braid group $\mathfrak{b}_{i}$ now represent the clockwise exchange of particle $i$ and $i+1$. The braid $\delta=\mathfrak{b}_{1} \ldots \mathfrak{b}_{n-1}$ becomes the rigid rotation by $2 \pi / n$ of the configuration in the clockwise direction, represented by the path $\delta(t)=e^{-2 \pi i t / n} z_{0}$. The braid $\Delta^{2}=\delta^{n}$ is the full twist, given by a clockwise rotation by $2 \pi$.

Let $k$ be coprime to $n$, and let $(g, \tau(g))=\left(R_{2 \pi a / k}, \sigma_{1}^{b},(1 / n k)\right) \in G_{\tau}=$ $D(n, \infty)$, the symmetry group of $u$, where $a n-b k=1$. Let $\gamma(t)=u(t / n k)$ for $t \in[0,1]$ (so $\gamma$ is the generator of $u$-Definition 4.3). Then $\gamma \in \Lambda^{g} X^{(n)}$, so it is natural to ask which connected component it belongs to. The components of $\Lambda^{g} X^{(n)}$ are indexed by the $P_{n}$-conjugacy classes of $\delta^{b} P_{n}<C B_{n}$ as described in the theorem above. A calculation involving the isomorphism of Theorem 5.4 shows that the corresponding braid is precisely $\delta^{b}$.

Since $b$ is coprime to $n$, it follows from results of [4] (see also [19]) that the centralizer of $\delta^{b}$ in $P_{n}$ is the cyclic group generated by $\Delta^{2}$. It then follows from Theorem 5.7 that the space of loops with symmetry $C(n, \infty)$ (or symmetry type $D(n, \infty))$ is homotopic to $S^{1}$, a fact which also follows from the direct argument in Example 5.11 below.

EXAMPLE 5.10. Consider the choreographies with three particles and fourfold rotational symmetry, so $G=C(3,4)$. Then $G \simeq \mathbb{Z}_{3} \times \mathbb{Z}_{4} \simeq \mathbb{Z}_{12}$, which is generated by

$$
(g, \tau(g))=\left(R_{3 \pi / 4}, \sigma_{1}^{-1}, \frac{1}{12}\right) \in \Gamma \times \mathrm{S}^{1}
$$

(see (2.3), with $a n-b k=1$ for $a=b=-1$ ), and we have that

$$
\pi_{1}^{G}\left(X^{(3)}, x\right) \simeq C B_{3} \times_{\alpha} \mathbb{Z}(1 / 4),
$$

where $\alpha(1)=\Delta^{2}$, and the set of connected components is in one-to-one correspondence with the $P_{3}$-conjugacy classes in the coset $\delta^{-1} P_{3} \subset B_{3}$ (in fact in $C B_{3}$ the cyclic braid group). 

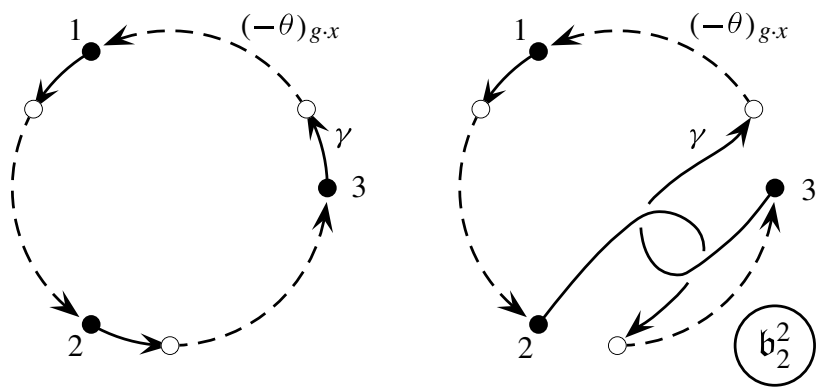

Figure 12. Two braids arising from loops with $D(3,4)$ symmetry. The first is the circular choreography, and the second the one depicted in Figure 4. The black dots represent the base point $z_{0} \in X^{(3)}$, and the open dots the point $\gamma(1)=g \cdot z_{0}$, where $g=\left(R_{-\pi / 2}, \sigma_{1}^{-1}\right)$, and $\sigma_{1}^{-1}=\left(\begin{array}{lll}1 & 3 & 2\end{array}\right)$. The solid curves represent $\gamma$ and the dashed ones $(-\theta)_{g \cdot x}$. (A break in the solid path in the second figure represents the one traversed later: in other words this is a top-down view of the motion, with time increasing downwards and the 'hidden' curve broken as usual.)

There are two examples of elements of $C(3,4)$ we have seen: the circular choreography with symmetry type $D(3, \infty)$, see Examples 1.3 and 5.9 above, and the choreography in Figure 4 (and note that of course $D(3,4)<C(3,4)$ ). The generators and braids of these are illustrated in Figure 12. The first of these corresponds to the $P_{3}$-conjugacy class containing $\delta^{-1}=\mathfrak{b}_{2}^{-1} \mathfrak{b}_{1}^{-1}$, as shown in the example above, while the second corresponds to the one containing $\mathfrak{b}_{2}^{2} \delta^{-1}=\mathfrak{b}_{2} \mathfrak{b}_{1}^{-1}$.

It was shown in the previous example that the $P_{3}$-centralizer of $\delta=\mathfrak{b}_{1} \mathfrak{b}_{2}$ is the subgroup of $P_{3}$ generated by $\Delta^{2}$, so that the set of loops with symmetry $C(3, \infty)$ has the homotopy type of a circle (as was shown explicitly in Example 5.9). On the other hand, the element $\mathfrak{b}_{2} \mathfrak{b}_{1}^{-1}$ is a pseudo-Anosov braid (by exclusion: it is neither periodic nor reducible [17]; or see [11, Section 15.1]), and it follows from work of González-Meneses and Wiest [19] that its $P_{3}$ centralizer is isomorphic to $\mathbb{Z}^{2}$ (in fact generated by $\left(\mathfrak{b}_{2} \mathfrak{b}_{1}^{-1}\right)^{3}$ and $\Delta^{2}$ ). Assuming that the functional is coercive (which occurs for example for the strong force; see Section 1.2), it follows by using Morse theoretic arguments that it must have at least two $S^{1}$-orbits of critical points on this component, for otherwise it would be homotopic to a circle.

Finally, we consider symmetry groups where the number of particles divides the order of symmetry of the curve, and show directly that such loops are always homotopic to the circular choreography, and indeed in the case of the Newtonian potential the only choreographic solution is the circular solution.

EXAMPLE 5.11. Suppose that $G=C(n, k / \ell)$, with $k$ a multiple of $n$. Then $c=(n, k)=n$, and the fixed point space $\operatorname{Fix}\left(K, X^{(n)}\right) \simeq X_{*}^{(1)}$, the punctured 
plane (see Lemma 3.1). Indeed, the position of the first particle determines the others, as they lie at the vertices of a regular $n$-gon centred at the origin. The motion will preserve this property, and so the solutions will be 'homothetic'. The fundamental group of this fixed point space is $\mathbb{Z}$, and the equivariant fundamental group $\pi_{1}^{G}\left(\mathbb{C}^{*}, x\right) \simeq \mathbb{Z} \times \mathbb{Z}_{k}$, with $\beta(p, q)=(p(\bmod n), q) \in G=\mathbb{Z}_{n} \times \mathbb{Z}_{k}$. The conjugacy is trivial, so there are countably many connected components (parameterized by $\pi_{1}\left(\mathbb{C}^{*}\right)=\mathbb{Z}$ ), and each connected component is homotopic to a circle.

More explicitly, let $u(t)=\left(z_{1}(t), \ldots, z_{n}(t)\right)$ be a choreography with symmetry $C(n, k / \ell)$, with $k=a n$. The symmetry implies in particular that, for all $t$, $z_{i}(t) / z_{j}(t)$ is never a positive real number. Define a new choreography $v$ homotopic to $u$ within the set of loops with symmetry $C(n, k / \ell)$ by projecting each point radially to the unit circle:

$$
v(t)=\left(\frac{z_{1}(t)}{\left|z_{1}(t)\right|}, \ldots, \frac{z_{n}(t)}{\left|z_{n}(t)\right|}\right) .
$$

This choreography involves all the points moving equally spaced around the unit circle, and so in fact has symmetry $D(n, \infty / \ell)$. It follows that every component of $C(n, k / \ell)$ with $n \mid k$ contains a loop with symmetry $D(n, \infty / \ell)$. Furthermore, each of the $\mathbb{Z}$ 's worth of connected components of $C(n, k / \ell)$ mentioned above contains the multiple coverings of the basic one.

As far as the dynamics is concerned, the potential function on $X^{(n)}$ restricts to a similar potential function on this fixed point space. In particular, if the potential is homogeneous of degree $d$, then the restriction is also homogeneous of degree $d$, and the resulting dynamics coincides with that for a single mass moving in the corresponding central force. For example, if the system is Newtonian $(d=-1)$, the restricted dynamics will involve the vertices of the regular $n$-gon moving in ellipses, parabolae, or hyperbolae. However, if the motion is not circular, then distinct vertices will move on distinct curves, so the only choreographic solution will be the circular motion. Similar results should be available for other homogeneous potentials.

In the next section, we extend this example by asking which components of $C(n, k)$ and $C(n, k / \ell)$ do not contain loops with greater symmetry.

\section{Adjacencies and components}

There is a familiar question in the application of topological methods to the calculus of variations which asks whether a given loop is homotopic to a loop which is multiply covered. If it is not, then existence theorems (such as existence of geodesics) guarantee there is a solution in the given homotopy class (connected component of loop space) which is not just a multiple covering of a 
simpler solution, so guaranteeing a 'new' solution. The answer is simply that a loop is not homotopic to a multiply covered loop if and only if the corresponding element of the fundamental group is primitive. An element of a group is said to be primitive if it cannot be written as a positive power of another element; this property is invariant under conjugacy.

From the symmetry perspective, even if there is no symmetry group on the configuration space $X$, there is always an $S^{1}$-action on the loop space $\Lambda X$ (we do not discuss the $\widehat{S}^{1}$ action in this section). A trivial loop is one with isotropy precisely $\mathrm{S}^{1}$. A loop has symmetry $\mathbb{Z}_{r}<\mathrm{S}^{1}$ if and only if it is $r$-times covered. So, if a loop $u$ has symmetry $\mathbb{Z}_{r}<\mathrm{S}^{1}$, then the curve $v: t \mapsto u(t / r)$, for $t \in[0,1]$, is also a loop, and the homotopy classes satisfy $[u]=[v]^{r}$, explaining what was said above. In particular, $u$ is freely homotopic to a multiply covered curve (that is, one with greater symmetry) if and only if $[u] \in \pi_{1}(X, x)$ is not a primitive element.

The natural extension of this question to the symmetric setting is to ask whether a particular connected component of the space of loops with symmetry $G$ contains loops with greater symmetry (of which multiple coverings are a particular case). We saw an instance of this in Example 5.11, where $G=C(n, k / \ell)$ with $n \mid k$ : all such components contain loops with symmetry $D(n, \infty / \ell)$.

In the context of choreographies, we saw in Section 2.5 that $C(n, k / \ell) \prec$ $C\left(n, k^{\prime}, \ell^{\prime}\right)$ if and only if $k \mid k^{\prime}$ and $\ell \equiv \ell^{\prime}(\bmod k)$. Some components of the loops with symmetry (at least) $C(n, k / \ell)$ contain loops with symmetry $C\left(n, k^{\prime}, \ell^{\prime}\right)$, and some do not. Similarly, if $n$ is odd, $C(n, 1) \prec C^{\prime}(n, 2)$, and the same question arises. In this section, we develop a method to determine which components do contain loops with greater symmetry and which do not, in terms of conjugacy classes in the equivariant fundamental group $\pi_{1}^{\Gamma}\left(X^{(n)}, x\right)$. If the action of $\Gamma$ on $X$ is free, this is a simple extension of the classical result above, the requirement being that the relevant element $(\gamma, g)$ be a primitive element of $\pi_{1}^{G}$-see Corollary 6.2 below.

6.1. Maximal symmetry type. Returning to the general context of actions of a group $\Gamma$ on a connected manifold $X$, suppose that a loop $u$ has symmetry equal to $G<\Gamma \times \mathrm{S}^{1}$, with $\tau(G)=\mathbb{Z}_{r}<\mathrm{S}^{1}$, and suppose that $g \in G$ is such that $\tau(g)=1 / r$. Such a $g$ is uniquely defined modulo $K=\operatorname{ker} \tau \triangleleft G$ and, moreover, since $u$ has isotropy $G, g \in N=N_{\Gamma}(K)$, the normalizer of $K$ in $\Gamma$. As usual, define in this case $\gamma(t)=u(t / r)$ for $t \in[0,1]$, the generator of $u$, so $u=\phi_{r}(\gamma)$ in the notation of (4.1). Then $\gamma \in \Lambda^{g} X^{K}$.

Let $C(u)$ denote the connected component of $\operatorname{Fix}(G, \Lambda X)$ containing $u$, or what is equivalent by Proposition 4.2, the connected component of $\Lambda^{g} X^{K}$ 
containing $\gamma$. The question is whether $C(u)$ contains any points with symmetry group strictly containing $G$. We say that the component $C(u)$ is of maximal symmetry type if there are no loops in $C(u)$ with symmetry strictly greater than $G$.

THEOREM 6.1. Suppose that the group $\Gamma$ acts on a manifold $X$, and let $u$ be a loop in $X$ with isotropy $G<\Gamma \times \mathrm{S}^{1}$, with $K=\operatorname{ker} \tau$ trivial, and let $\gamma \in \Lambda^{g} X$ be a generator of $u$. The connected component $C(u)$ of $\operatorname{Fix}(G, \Lambda X)$ containing $u$ is of maximal symmetry type if and only if both the following conditions hold:

(1) $(\gamma, g) \in \pi_{1}^{\Gamma}(X, x)$ is a primitive element; and

(2) there does not exist a nontrivial isotropy subgroup $K$ of the $\Gamma$-action on $X$ for which $(\gamma, g) \in \pi_{1}^{N}\left(X^{K}, x^{\prime}\right)$, where $N=N_{\Gamma}(K)$ and $x^{\prime} \in X^{K}$.

More generally, the same is true if $u \in \Lambda Y$ for $Y=X^{K_{0}}$, with $K_{0}=\operatorname{ker} \tau \triangleleft G$ nontrivial, provided that $\Gamma$ is replaced by $\Gamma_{0}:=N_{\Gamma}\left(K_{0}\right) / K_{0}, X$ is replaced by $Y$, and, for condition (2), $K$ strictly contains $K_{0}, x$ and $x^{\prime}$ should be in the same connected component of $X^{K_{0}}$, and $N$ is replaced by

$$
N^{\prime}=\left(N_{\Gamma}\left(K_{0}\right) \cap N_{\Gamma}(K)\right) / K_{0} .
$$

Condition (2) implicitly uses the identification of $\pi_{1}^{N}\left(X^{K}, x^{\prime}\right)$ with its image in $\pi_{1}^{\Gamma}(X, x)$ (since $X^{K} \subset X$ and $\left.N<\Gamma\right)$. More generally, it uses the identification of $\pi_{1}^{N^{\prime}}\left(X^{K}, x\right)$ with its image in $\pi_{1}^{\Gamma_{0}}\left(X^{K_{0}}, x\right)$. Note that this condition (2) is trivially satisfied if $K$ is a maximal isotropy subgroup of the $\Gamma$-action on $X$.

In the more general version of condition (1), the group $\pi_{1}^{\Gamma_{0}}(Y, x)$ arises from the natural action of $N_{\Gamma}\left(K_{0}\right) / K_{0}$ on $Y=X^{K_{0}}$, and is isomorphic to the quotient

$$
\mathbf{1} \longrightarrow K_{0} \longrightarrow \pi_{1}^{N_{\Gamma}\left(K_{0}\right)}(Y, x) \longrightarrow \pi_{1}^{\Gamma_{0}}(Y, x) \longrightarrow \mathbf{1},
$$

where the first inclusion is $k \mapsto(\bar{x}, k)$, where $\bar{x}$ is the constant loop at $x$.

Proof. We prove the 'basic' version; the more general version follows by considering the $\Gamma_{0}$-action on $X^{K_{0}}$.

First, we prove the 'only if' statement. Suppose first that $(\gamma, g) \in \pi_{1}^{\Gamma}(X, x)$ is not primitive, so there is a $(\delta, h) \in \pi_{1}^{\Gamma}(X, x)$ and $p>1$ such that $(\gamma, g)=(\delta, h)^{p}$. Let $v$ be the loop corresponding to $\delta$. Then $v$ is homotopic to $u$ in $\operatorname{Fix}(G, \Lambda X)$, so $C(u)$ is not maximal. Second, suppose that condition (2) is violated. Since $X$ is connected, the groups $\pi_{1}^{\Gamma}(X, x)$ and $\pi_{1}^{\Gamma}\left(X, x^{\prime}\right)$ are isomorphic, so let $\left(\gamma^{\prime}, g\right)$ be the corresponding element in the latter group (same $g$ ). Then there is a $(\delta, h) \in \pi_{1}^{N}\left(X^{K}, x^{\prime}\right)$ such that $\left(\gamma^{\prime}, g\right)=(\delta, h)$ ( $h$ is only defined modulo $K$, but we can choose $h=g$ ). It follows that $u$ and $v$ are homotopic.

For the converse, suppose that the component $C(u)$ is not of maximal symmetry type, and let $v \in C(u)$ be a loop with greater symmetry. That is, $v \in$ 
Fix $(H, \Lambda X) \cap C(u)$ for some group $H<\Gamma \times \mathrm{S}^{1}$ with $H \supsetneqq G$. Let $\tau^{\prime}: H \rightarrow \mathrm{S}^{1}$ be the projection, and let $K$ be the kernel. If $K$ is nontrivial, then condition (2) fails.

On the other hand, if $K$ is trivial, then the image of $\tau^{\prime}$ is a subgroup of $\mathrm{S}^{1}$ strictly containing $\tau(G)$, so has order $p r$ for some integer $p>1$. Let $h$ satisfy $\tau^{\prime}(h)=1 / p r$, and define $\delta(t)=v(t / p r)$ for $t \in[0,1]$. Then $\delta \in \Lambda^{h} X$, and $(\gamma, g)=(\delta, h)^{p}$, so that $(\gamma, h)$ is not primitive.

As mentioned above, if the action of $\Gamma$ on $X$ is free, the theorem simplifies considerably.

Corollary 6.2. Suppose that the action of $\Gamma$ on $X$ is free, and let $u$ be a loop with symmetry $G<\Gamma \times S^{1}$. The connected component $C(u)$ of $\operatorname{Fix}(G, \Lambda X)$ is of maximal symmetry type if and only if the corresponding element $(\gamma, g) \in$ $\pi_{1}^{\Gamma}(X, x)$ is primitive, where $\gamma$ is the generator of $u$.

6.2. Application to choreographies. Since we know from Theorem 2.3 all the isotropy subgroups of $\Gamma \times \mathrm{S}^{1}$ appearing for choreographies, we can simplify the application of the theorem above. There are two types of case to consider. The first, more direct and only for odd $n$, is that $C(n, 1)=\mathfrak{C}_{n} \prec C^{\prime}(n, 2)$, and the other, which involves many subcases, is that $C(n, k / \ell) \prec C\left(n, k^{\prime} / \ell^{\prime}\right)$ if and only if $k \mid k^{\prime}$ and $\ell^{\prime} \equiv \pm \ell(\bmod k)$.

Suppose first that $n$ is odd and that $u$ has symmetry $C(n, 1)$. By Theorem 5.7, the set of connected components of $C(n, 1)$ is in one-to-one correspondence with the $P_{n}$-conjugacy classes in the coset $\delta^{-1} P_{n} \subset C B_{n}$ (the cyclic braids are defined in Equation (5.7)). The connected component $C(u)$ containing $u$ therefore corresponds to the $P_{n}$-conjugacy class containing $\delta^{-1} p_{u}$ for some $p_{u} \in P_{n}$.

Suppose that $v \in C(u)$ has symmetry $C^{\prime}(n, 2)$, and let $\eta(t)=v(t / 2 n)$, so that $\eta \in \Lambda^{\left(\kappa, \sigma_{2}\right)} X^{(n)}$ (see Section 2.1 for notation). Since there is no rotational part to this symmetry group, the relevant equivariant fundamental group is

$$
\pi_{1}^{\mathbb{Z}_{2} \times \Sigma_{n}}\left(X^{(n)}, x\right) \simeq C B_{n} \rtimes \mathbb{Z}_{2},
$$

with product $\left(\mathfrak{b}_{1}, \kappa\right)\left(\mathfrak{b}_{2}, g\right)=\left(\mathfrak{b}_{1} \overline{\mathfrak{b}}_{2}, \kappa g\right)$ for $g \in\{I, \kappa\}$, and $\overline{\mathfrak{b}}$ is the braid obtained from $\mathfrak{b}$ by changing all overcrossings to undercrossings and vice versa (see Theorem 5.4). The map $\phi_{2}: \Lambda^{\left(\kappa, \sigma_{2}\right)} X^{(n)} \rightarrow \Lambda^{\mathfrak{c}} X^{(n)}$ is

$$
\left(\gamma,\left(\kappa, \sigma_{2}\right)\right) \longmapsto(\gamma * \bar{\gamma}, \mathfrak{c}) .
$$

Furthermore, connected components correspond to twisted conjugacy classes, and indeed, under this map, for arbitrary $r \in P_{n}$,

$$
r * \gamma * \bar{r}^{-1} \longmapsto\left(r * \gamma * \bar{r}^{-1}\right) *\left(\bar{r} * \bar{\gamma} * r^{-1}\right)=r *(\gamma * \bar{\gamma}) * r^{-1},
$$


up to homotopy, so mapping connected components to connected components (as it must!). Moreover, with $\left(\gamma,\left(\kappa, \sigma_{2}\right)\right)$ corresponding to $\left(\mathfrak{b}_{0}, \kappa\right) \in B_{n} \rtimes \mathbb{Z}_{2}$, we have that $\mathfrak{b}_{0}=\delta^{\prime} p$ for some pure braid $p$ (see Theorem 5.7). The corresponding braid under $\phi_{2}$ is $\mathfrak{b}_{0} \overline{\mathfrak{b}}_{0}=\delta^{\prime} p \overline{\delta^{\prime}} \bar{p}=\delta^{\prime} \overline{\delta^{\prime}} p^{\prime} \bar{p}=\delta p^{\prime \prime}$, as required for braids with $C(n, 1)$ symmetry. Thus we have, for odd $n$, the following.

PROpOSITION 6.3. A loop $u$ with symmetry $C(n, 1)$ is homotopic to a loop with symmetry $C^{\prime}(n, 2)$ if and only if the corresponding element $\mathfrak{b}$ of the braid group can be written as $\mathfrak{b}=\mathfrak{b}_{0} \overline{\mathfrak{b}_{0}}$.

Note that $\pi(\mathfrak{b})=\pi\left(\mathfrak{b}_{0}\right)^{2}=\sigma_{1}$, which implies that $\pi\left(\mathfrak{b}_{0}\right)=\sigma_{2}\left(\sigma_{2}\right.$ is the unique square root of $\sigma_{1}$ in $S_{n}$ ), so any $\mathfrak{b}_{0}$ satisfying $\mathfrak{b}=\mathfrak{b}_{0} \overline{\mathfrak{b}_{0}}$ corresponds to a loop with symmetry $C^{\prime}(n, 2)$.

On the other hand, as pointed out earlier, the exponent sum satisfies $\chi(\overline{\mathfrak{b}})=$ $-\chi(\mathfrak{b})$. It follows that $\chi(\mathfrak{b} \bar{b})=0$, and consequently the braid associated to any component of the set of choreographies containing loops with $C^{\prime}(n, 2)$ symmetry must have zero exponent sum.

An open question arising here is whether, for $g^{\prime}$ the generator of $C^{\prime}(n, 2)$, the map

$$
\pi_{0}\left(\Lambda^{g^{\prime}} X^{(n)}\right) \longrightarrow \pi_{0}\left(\Lambda^{\mathrm{c}} X^{(n)}\right)
$$

is injective. Failure of this would amount to the existence of two braids $\mathfrak{b}, \mathfrak{b}^{\prime} \in$ $\delta^{\prime} P_{n}$ in distinct twisted conjugacy classes, for which $\mathfrak{b} \overline{\mathfrak{b}}$ is conjugate to $\mathfrak{b}^{\prime} \overline{\mathfrak{b}}^{\prime}$. This would have implications for the number of critical points of the action functional in a connected component of $C(n, 1)$, as there would be at least two critical points in that component with symmetry (conjugate to) $C^{\prime}(n, 2)$.

Now consider the case $C(n, 1) \prec C(n, k / \ell)$, with $(n, k)=1$. Since there are no fixed points for the $\Gamma$-action, we are in the setting of Corollary 6.2. Let $g=\left(R_{2 \pi a \ell / k}, \sigma_{1}^{b}\right)$, and $\delta \in \Lambda^{g} X^{(n)}$, where as usual $a n-b k=1$. Then the map $\phi_{k}: \Lambda^{g} X^{(n)} \rightarrow \Lambda^{\mathrm{c}} X^{(n)}$, as in Equation (4.1), and, at the level of homotopy,

$$
\phi_{k}(\eta, g)=(\eta, g)^{k}=(\gamma, \mathfrak{c}) \text {, }
$$

with $\gamma=\phi_{k}(\eta)$. Representing $(\eta, g) \in \pi_{1}^{\Gamma}\left(X^{(n)}, x\right)$ as $\left[\mathfrak{b}_{0}, a \ell / k\right] \in B_{n} \times_{\alpha} \mathbb{R}$ and $(\gamma, \mathfrak{c})$ as $[\mathfrak{b}, 0]$, we require that $\left[\mathfrak{b}_{0}, a \ell / k\right]^{k}=[\mathfrak{b}, 0]$. This becomes

$$
\left[\mathfrak{b}_{0}^{k}, a \ell\right]=[\mathfrak{b}, 0],
$$

so that $\mathfrak{b}=\Delta^{-2 a \ell} \mathfrak{b}_{0}^{k}$. This implies the following.

PRoposition 6.4. A loop $u$ with symmetry $C(n, 1)$ and corresponding braid $\mathfrak{b} \in \delta^{-1} P_{n}$ is homotopic to a loop with symmetry $C(n, k / \ell)$ with $(n, k)=1$ if and only if $\Delta^{2 a \ell} \mathfrak{b}$ has a $k$ th root, where an $\equiv 1(\bmod k)$. 
Having a $k$ th root means of course that it can be written as $\Delta^{2 a \ell} \mathfrak{b}=\mathfrak{b}_{0}^{k}$ for some braid $\mathfrak{b}_{0}$. Since $\chi\left(\Delta^{2}\right)=n(n-1)$, this requires $2 a \ell n(n-1)+\chi(\mathfrak{b})$ to be a multiple of $k$, and hence the exponent sum satisfies

$$
\chi(\mathfrak{b}) \equiv-(n-1) \ell(\bmod k) .
$$

This restricts the possible values of $k, \ell$. We aim to consider the full question, allowing for the core, in a future paper.

Finally, we mention a result of González-Meneses [17], who shows that the $k$ th root of a braid, if it exists, is unique up to conjugacy in $B_{n}$. Moreover, if the braid is of pseudo-Anosov type, then a $k$ th root, if it exists, is unique. (The type of $\mathfrak{b}$ coincides with the type of $\Delta^{2} \mathfrak{b}$, as is readily checked.) This suggests that the map

$$
\pi_{0}\left(\Lambda^{g} X^{(n)}\right) \longrightarrow \pi_{0}\left(\Lambda^{\mathrm{c}} X^{(n)}\right)
$$

may be injective. However, the conjugacy in [17] is by all possible elements of $B_{n}$, while $\pi_{0}$ is determined by conjugacy with respect to elements of $P_{n}$. So this is inconclusive, but again would have repercussions for estimates of numbers of critical points.

\section{Acknowledgements}

We would like to thank Mark Roberts, Jelena Grbic, and Peter Rowley for helpful discussions, and Dan Gries for creating the HTML5/Javascript code which displays the animations on the website [27]. We would also like to thank the two anonymous referees whose suggestions have helped improve the presentation. The second author was supported during her $\mathrm{PhD}$ studies by Forrest Recruitment, and she would like to express her gratitude to John and Stephanie Forrest for their support, and the interest they took in this research.

\section{Supplementary materials}

Supplementary materials are available at http://dx.doi.org/10.1017/fms.2013.5.

\section{References}

[1] E. Barrabés, J. M. Cors, C. Pinyol and J. Soler, 'Hip-hop solutions of the $2 n$-body problem', Celestial Mech. Dynam. Astronom. 95 (2006), 55-66.

[2] V. Barutello, D. Ferrario and S. Terracini, 'Symmetry groups of the planar 3-body problem and action-mimizing trajectories', Arch. Ration. Mech. Anal. 190 (2008), 189-226.

[3] V. Barutello and S. Terracini, 'Action minimizing orbits in the $n$-body problem with simple choreography constraint', Nonlinearity 17 (2004), 2015-2039. 
[4] D. Bessis, F. Digne and J. Michel, 'Springer theory in braid groups and the Birman-Ko-Lee monoid', Pacific J. Math. 205(2) (2002), 287-309.

[5] A. Chenciner, 'A note by Poincaré', Regul. Chaotic Dyn. 10(2) (2005), 119-128.

[6] A. Chenciner, 'Poincaré and the three-body problem', Sémin. Poincaré XVI (2012), 45-133.

[7] A. Chenciner, J. Gerver, R. Montgomery and C. Simó, 'Simple choreographic motions of $N$ bodies: a preliminary study', in Geometry, Mechanics, and Dynamics (Springer, New York, 2002), 287-308.

[8] A. Chenciner and R. Montgomery, 'A remarkable periodic solution of the three-body problem in the case of equal masses', Ann. of Math. (2) 152 (2000), 881-901.

[9] A. Chenciner and A. Venturelli, 'Minima de l'intégrale d'action du problème newtonien de 4 corps de masses égales dans $\mathbb{R}^{3}$ : orbites hip-hop', Celestial Mech. Dynam. Astronom. 77(2) (2000), 139-152.

[10] E. Fadell and L. Neuwirth, 'Configuration spaces', Math. Scand. 10 (1962), 111-118.

[11] B. Farb and D. Margalit, A Primer on Mapping Class Groups, Princeton Mathematical Series, 49 (Princeton University Press, Princeton, NJ, 2012).

[12] D. Ferrario, 'Transitive decomposition of symmetry groups for the $n$-body problem', Adv. Math. 213(2) (2007), 763-784.

[13] D. L. Ferrario and S. Terracini, 'On the existence of collisionless equivariant minimizers for the classical $n$-body problem', Invent. Math. 155(2) (2004), 305-362.

[14] R. Fox and L. Neuwirth, 'The braid groups', Math. Scand. 10 (1962), 119-126.

[15] G. Fusco, G. F. Gronchi and P. Negrini, 'Platonic polyhedra, topological constraints and periodic solutions of the classical N-body problem', Invent. Math. 185(2) (2011), 283-332.

[16] M. Golubitsky and I. Stewart, 'Hopf bifurcation in the presence of symmetry', Arch. Ration. Mech. Anal. 87 (1985), 107-165.

[17] J. González-Meneses, 'The $n$th root of a braid is unique up to conjugacy', Algebr. Geom. Topol. 3 (2003), 1103-1118, electronic.

[18] J. González-Meneses and E. Ventura, (2011) Twisted conjugacy in braid groups. ArXiv 1104.5690 [MATH.GT].

[19] J. González-Meneses and B. Wiest, 'On the structure of the centralizer of a braid', Ann. Sci. Éc. Norm. Supér. (4) 37 (2004), 729-757.

[20] W. B. Gordon, 'Conservative dynamical systems involving strong forces', Trans. Amer. Math. Soc. 204 (1975), 113-135.

[21] V. W. Guillemin and S. Sternberg, 'Supersymmetry and equivariant de Rham theory', in Mathematics Past and Present (Springer, Berlin, 1999), With an appendix containing two reprints by Henri Cartan [MR0042426 (13,107e); MR0042427 (13,107f)].

[22] A. Hatcher, Algebraic Topology (Cambridge University Press, Cambridge, 2002).

[23] J. Jost and X. Li-Jost, Calculus of Variations, Cambridge Studies in Advanced Mathematics, 64 (Cambridge University Press, Cambridge, 1998).

[24] C. Kassel and V. Turaev, Braid Groups, Graduate Texts in Mathematics, vol. 247 (Springer, New York, 2008).

[25] W. Klingenberg, Lectures on Closed Geodesics, Grundlehren der Mathematischen Wissenschaften, 230 (Springer, Berlin, 1978).

[26] C. McCord, J. Montaldi, M. Roberts and L. Sbano, 'Relative periodic orbits of symmetric Lagrangian systems', in EQUADIFF 2003 (World Sci. Publ, Hackensack, NJ, 2005), $482-493$. 
[27] J. Montaldi, http://www.maths.manchester.ac.uk/jm/Choreographies.

[28] J. A. Montaldi, R. M. Roberts and I. N. Stewart, 'Periodic solutions near equilibria of symmetric Hamiltonian systems', Philos. Trans. R. Soc. Lond. Ser. A 325 (1988), 237-293.

[29] R. Montgomery, 'The $N$-body problem, the braid group, and action-minimizing periodic solutions', Nonlinearity 11 (1998), 363-376.

[30] R. Montgomery, 'A new solution to the three-body problem', Not. Amer. Math. Soc. 48 (2001), 471-481.

[31] C. Moore, 'Braids in classical dynamics', Phys. Rev. Lett. 70(24) (1993), 3675-3679.

[32] R. Palais, 'The principle of symmetric criticality', Comm. Math. Phys. 69(1) (1979), 19-30.

[33] F. Rhodes, 'On the fundamental group of a transformation group', Proc. Lond. Math. Soc.

(3) 16 (1966), 635-650.

[34] C. Simó, 'New families of solutions in $n$-body problems', in European Congress of Mathematics, Vol. I (2001).

[35] C. Simó, 'Periodic orbits of the planar $n$-body problem with equal masses and all bodies on the same path', in The Restless Universe (eds. B. A. Steves and A. J. Maciejewski) (2001), 265-284.

[36] E. H. Spanier, Algebraic Topology (Springer, New York, 1981), Corrected reprint.

[37] K. Steckles, Loop Spaces and Choreographies in Dynamical Systems. Ph.D. Thesis, University of Manchester, 2011. http://eprints.ma.man.ac.uk/1807.

[38] I. Stewart, 'Symmetry methods in collisionless many-body problems', J. Nonlinear Sci. 6 (1996), 543-563.

[39] S. Terracini, 'On the variational approach to the periodic $n$-body problem', Celestial Mech. Dynam. Astronom. 95 (2006), 3-25.

[40] V. V. Vershinin, 'Braid groups and loop spaces', Uspekhi Mat. Nauk 54(2(326)) (1999), $3-84$. 